\title{
On multiple ergodicity of affine cocycles over irrational rotations
}

\author{
Jean-Pierre Conze and Agata Piękniewska
}

September 11, 2018

\begin{abstract}
Let $T_{\alpha}$ denote the rotation $T_{\alpha} x=x+\alpha(\bmod 1)$ by an irrational number $\alpha$ on the additive circle $\mathbb{T}=[0,1)$. Let $\beta_{1}, \ldots, \beta_{d}$ be $d \geq 1$ parameters in $[0,1)$. One of the goals of this paper is to describe the ergodic properties of the cocycle (taking values in $\mathbb{R}^{d+1}$ ) generated over $T_{\alpha}$ by the vectorial function $\Psi_{d+1}(x):=$ $\left(\varphi(x), \varphi\left(x+\beta_{1}\right), \ldots, \varphi\left(x+\beta_{d}\right)\right)$, with $\varphi(x)=\{x\}-\frac{1}{2}$.

It was already proved in [LeMeNa03] that $\Psi_{2}$ is regular for $\alpha$ with bounded partial quotients. In the present paper we show that $\Psi_{2}$ is regular for any irrational $\alpha$. For higher dimensions, we give sufficient conditions for regularity. While the case $d=2$ remains unsolved, for $d=3$ we provide examples of non-regular cocycles $\Psi_{4}$ for certain values of the parameters $\beta_{1}, \beta_{2}, \beta_{3}$.

We also show that the problem of regularity for the cocycle $\Psi_{d+1}$ reduces to the regularity of the cocycles of the form $\Phi_{d}=\left(1_{\left[0, \beta_{j}\right]}-\beta_{j}\right)_{j=1, \ldots, d}$ (taking values in $\left.\mathbb{R}^{d}\right)$. Therefore, a large part of the paper is devoted to the classification problems of step functions with values in $\mathbb{R}^{d}$.
\end{abstract}

\section{Contents}

1 Introduction

2 Preliminaries

2.1 Irrational rotations . . . . . . . . . . . . . . . . 3

2.2 Essential values of cocycles taking values in Abelian groups . . . . . . . ฤ ฤ

2.3 Essential values of cocycles taking values in $\mathbb{R}^{d} \ldots \ldots$. . . . . . . . 12

3 Step cocycles over an irrational rotation $\quad 14$

3.1 Rational step cocycles . . . . . . . . . . . . . . 16

3.2 Reduction in the bounded type case . . . . . . . . . . . . . . 18 
3.3 The case $\left\|q_{n} \beta_{j}\right\| \rightarrow 0 \ldots \ldots \ldots$

3.4 Well separated discontinuities, clusters of discontinuities . . . . . . 21

3.5 On the regularity of $\Phi_{d}, d=1,2,3 \ldots \ldots \ldots \ldots \ldots$

4 Application to affine cocycles $\quad 29$

4.1 Reduction to a step function . . . . . . . . . . . . . . . . 2 29

4.2 Small values of $d=1,2,3$ (and $\left.\Psi_{d+1}\right) \ldots \ldots \ldots \ldots$

4.3 Ergodicity is generic . . . . . . . . . . . . . . . . 31

\section{Introduction}

Denote by $\mathbb{T}=[0,1)$ the additive circle. Let $\alpha \in(0,1)$ be an irrational number and $T_{\alpha}$ stand for the corresponding circle rotation: $T_{\alpha} x=x+\alpha$. The meaning of $\alpha$ being fixed, throughout the paper, we will write $T$ instead of $T_{\alpha}$ (except for Sections 2.2 and 2.3).

Let $\beta_{1}, \ldots, \beta_{d}$ be $d \geq 1$ parameters in $[0,1)$. We consider the cocycle generated over $T$ by the vectorial function

$$
\Psi_{d+1}(x):=\left(\psi(x), \psi\left(x+\beta_{1}\right), \ldots, \psi\left(x+\beta_{d}\right)\right), \text { with } \psi(x)=\{x\}-\frac{1}{2} .
$$

This cocycle takes values in $\mathbb{R}^{d+1}$ and one of the goals of this paper is to describe its ergodic properties. Namely, we are mostly interested whether or not $\Psi_{d+1}$ is regular (that is, cohomologous to a "smaller" cocycle which is ergodic, see Section 2.2 for the precise meaning of regularity). It is well known that $\Psi_{1}: \mathbb{T} \rightarrow \mathbb{R}$ is ergodic for each irrational $\alpha$, but for $d \geq 1$ the problem of regularity is unsolved. As for applications in ergodic theory, or more precisely in the theory of joinings, an importance of regularity of cocycles of the form (11) has been shown in [LeMeNa03]. Indeed, Theorem 3 therein gives the full description of all ergodic self-joinings for so called Rokhlin extensions given by regular cocycles. In particular, it is shown in LeMeNa03 that $\Psi_{2}$ is regular whenever $\alpha$ has bounded partial quotients. In the present paper we show that $\Psi_{2}$ is regular without the assumption of boundedness on the partial quotients of $\alpha$. For higher dimensions $(d \geq 2)$, we give sufficient conditions for regularity. While the case $d=2$ we leave unsolved, for $d=3$ we give examples of non-regular cocycles $\Psi_{4}$ for certain values of the parameters $\beta_{j}, j=1,2,3$. In Section 4.3, we show that the cocycle (11) is ergodic for a generic choice (in the measure-theoretic and the topological sense) of parameters $\beta_{1}, \ldots, \beta_{d}, d \geq 2$.

One of our basic tools is Theorem 4.1 below. It states that the group of essential values of $\Psi_{d+1}$ contains the diagonal subgroup $\Delta_{d+1}=\{(t, \ldots, t): t \in \mathbb{R}\} \subset \mathbb{R}^{d+1}$. It follows that the problem of regularity for the cocycle $\Psi_{d+1}$ is reduced to the regularity of the cocycles of the form $\Phi_{d}=\left(1_{\left[0, \beta_{j}\right)}-\beta_{j}, j=1, \ldots, d\right)$. Note that by taking linear combinations of cocycles of the form $\Phi_{d}$ we can get every step cocycle. Therefore, we devote a large part of the paper to the problem of classification of steps functions with values in $\mathbb{R}^{d}$. 
The problem of ergodicity or regularity of step functions, mainly in the one dimensional case, has been broadly studied in the literature, for instance see: [Or83], Pa90], [Fr00], [LePaVo96], Co09], [Zh10]; note that in Corollary [3.9 we generalize the main result of [Zh10]. We would like to emphasize that the methods presented in the paper, in large part (see Section 3), seem to be new and they contribute to a better understanding of the problem of regularity of general vectorial cocycles $\Phi: \mathbb{T} \rightarrow \mathbb{R}^{d}$ over irrational rotations.

\section{Preliminaries}

\subsection{Irrational rotations}

Let us recall some basic facts about continued fractions (e.g. [Kh]). Let $\left[0 ; a_{1}, \ldots, a_{n}, \ldots\right]$ be the continued fraction representation of $\alpha$, and let $\left(p_{n} / q_{n}\right)_{n>-1}$ be the sequence of its convergents. The integers $p_{n}$ (resp. $q_{n}$ ) are the numerators (resp. denominators) of $\alpha$. We have $p_{-1}=1, p_{0}=0, q_{-1}=0, q_{0}=1$, and for $n \geq 1$ :

$$
p_{n}=a_{n} p_{n-1}+p_{n-2}, q_{n}=a_{n} q_{n-1}+q_{n-2},(-1)^{n}=p_{n-1} q_{n}-p_{n} q_{n-1} .
$$

As usual the fractional part of $u \in \mathbb{R}$ is $\{u\}=u-[u]$, where $[u]$ is the integral part of $u$. For $u \in \mathbb{R}$, set $\|u\|=\inf _{n \in \mathbb{Z}}|u-n|=\min (\{u\}, 1-\{u\})$. Then for any integer $M$ we have $\|M u\| \leqslant|M|\|u\|$. Note that $\|\cdot\|$ introduces a translation invariant distance on $\mathbb{T}$.

We have for $n \geq 0,\left\|q_{n} \alpha\right\|=(-1)^{n} \theta_{n}$ with $\theta_{n}=q_{n} \alpha-p_{n}$, and moreover

$$
\begin{aligned}
1 & =q_{n}\left\|q_{n+1} \alpha\right\|+q_{n+1}\left\|q_{n} \alpha\right\| \\
\frac{1}{q_{n+1}+q_{n}} & \leq\left\|q_{n} \alpha\right\| \leq \frac{1}{q_{n+1}}=\frac{1}{a_{n+1} q_{n}+q_{n-1}} \\
\left\|q_{n} \alpha\right\| & \leq\|k \alpha\|, \text { for } 1 \leq|k|<q_{n+1} .
\end{aligned}
$$

An irrational $\alpha$ is said to be of bounded type if the sequence $\left(a_{n}\right)$ is bounded.

We need some preliminary lemmas on the diophantine properties of $\alpha$.

Lemma 2.1. 1) Let $p, q$ be two coprime positive integers and $\theta=q\left(\alpha-\frac{p}{q}\right)$ with $|\theta|<\frac{1}{q}$. When $\theta>0$, each interval $\left[\frac{j}{q}, \frac{j+1}{q}\right), 0 \leq j \leq q-1$ contains one (and only one) number of the form $\{k \alpha\}$, with $0 \leq k \leq q-1$. When $\theta<0$ the same is true for $j=1, \ldots, q-2$; there are two points $k \alpha$ (one for $k=0$ ) in $\left[0, \frac{1}{q}\right.$ ) and no such a point in $\left[\frac{q-1}{q}, 1\right)$.

2) For each $x \in \mathbb{T}$ the distance between two consecutive elements of the set $\{\{x+k \alpha\}$ : $k=0, \ldots, q-1\}$ is $<\frac{2}{q}$.

3) There are at most two elements of the set $\{\{x+k \alpha\}: k=0, \ldots, q-1\}$ in any interval on the circle of length $\frac{1}{q}$ (hence at most four such elements in any interval of length $\frac{2}{q}$ ).

4) If additionally $q=q_{n}$, the distance between two consecutive elements of the set $\{\{x-$ $k \alpha\}: k=0, \ldots, q\}$ is $>\frac{1}{2 q_{n}}$. 
Proof. The map $k \rightarrow k p \bmod q=j(k)$ is a bijection of $\{0,1, \ldots, q-1\}$ onto itself. If $\theta>0$, then $\{k \alpha\}=\left\{k\left(\frac{p}{q}+\frac{\theta}{q}\right)\right\}=\frac{j(k)}{q}+\frac{k \theta}{q}$ is at distance $\frac{k \theta}{q}<\frac{1}{q}$ from $\frac{j(k)}{q}$, hence it is in the interval $\left[\frac{j(k)}{q}, \frac{j(k)+1}{q}\right)$. The proof is similar if $\theta<0$. Hence the first assertion follows. Assertion 2) is true for $x=0$ by 1 ); hence, because the distance is invariant by translations, it is true for any $x \in \mathbb{T}$.

For 3), suppose that there are $\left\{x+k_{1} \alpha\right\}<\left\{x+k_{2} \alpha\right\}<\left\{x+k_{3} \alpha\right\}$ distinct in an interval of length $<1 / q$. We have $\frac{\ell}{q} \leq\left\{k_{1} \alpha\right\}<\left\{k_{2} \alpha\right\}<\left\{k_{3} \alpha\right\}<\frac{\ell+2}{q}$, for some $\ell$. Either $\left[\frac{\ell}{q}, \frac{\ell+1}{q}\right)$ or $\left[\frac{\ell+1}{q}, \frac{\ell+2}{q}\right)$ contains two points of the set $\{\{k \alpha\}: 0 \leq k<q-1\}$, which clearly contradicts 1).

4) We have the following

$$
\frac{1}{2 q_{n}} \leq \frac{1}{q_{n}+q_{n-1}} \leq\left\|q_{n-1} \alpha\right\| \leq\|j \alpha\|, \forall j,|j|<q_{n}
$$

and the assertion follows.

The first assertion of Lemma 2.1 implies easily the well-known Denjoy-Koksma inequality: let $\varphi$ be a centered function of bounded variation $V(\varphi)$ and $p / q$ a rational number (in lowest terms) such that $|\alpha-p / q|<1 / q^{2}$, then

$$
\left|\sum_{\ell=0}^{q-1} \varphi(x+\ell \alpha)\right| \leq V(\varphi)
$$

Indeed, let us consider the case $\theta>0$ (the proof is analogous when $\theta<0$ ). We can assume $x=0$. For $j=0, \ldots, q-1$, there is one and only one point $\left\{k_{j} \alpha\right\}$ of the set $(\{k \alpha\}, k=0, \ldots, q-1)$ in $I_{j}:=\left[\frac{j}{q}, \frac{j+1}{q}\right)$. Therefore, since $\int \varphi d t=0$, we have:

$$
\begin{aligned}
\left|\sum_{j=0}^{q-1} \varphi(j \alpha)\right| & =\left|\sum_{j=0}^{q-1} \varphi\left(\left\{k_{j} \alpha\right\}\right)-q \int \varphi(t) d t\right|=\left|\sum_{j=0}^{q-1} q \int_{j / q}^{(j+1) / q}\left[\varphi\left(\left\{k_{j} \alpha\right\}\right)-\varphi(t)\right] d t\right| \\
& \leq q \sum_{j=0}^{q-1} \int_{j / q}^{(j+1) / q}\left|\varphi\left(\left\{k_{j} \alpha\right\}\right)-\varphi(t)\right| d t \leq \sum_{j=0}^{q-1} \operatorname{Var}\left(\varphi, I_{j}\right)=\operatorname{Var}(\varphi,[0,1)) .
\end{aligned}
$$

Lemma 2.2. 1) If there exists $n_{0}$ such that $\inf _{0 \leq|j|<q_{n}}\|\beta-j \alpha\|<\frac{1}{2}\left\|q_{n+1} \alpha\right\|, \forall n \geq n_{0}$, then $\beta \in \mathbb{Z} \alpha+\mathbb{Z}$.

2) Suppose $\alpha$ of bounded type.

a) If $\beta \notin \mathbb{Z} \alpha+\mathbb{Z}$, then there exist $c>0$ and an increasing sequence $\left(n_{k}\right)$ such that, for every $k \geq 1,\|\beta-j \alpha\| \geq c / q_{n_{k}}$, for $0 \leq|j| \leq q_{n_{k}}$.

b) If $\beta=\frac{t}{r} \alpha+\frac{u}{s} \in(\mathbb{Q} \alpha+\mathbb{Q}) \backslash(\mathbb{Z} \alpha+\mathbb{Z})$, then there exists $c>0$ such that $\|\beta-j \alpha\| \geq c / q_{n}$, for $0 \leq|j| \leq q_{n}(n \geq 1)$.

Proof. 1) For each $n \geq 1$, consider the family of intervals $I_{n}^{j}=\left[\{j \alpha\}-\frac{1}{2}\left\|q_{n+1} \alpha\right\|,\{j \alpha\}+\right.$ $\left.\frac{1}{2}\left\|q_{n+1} \alpha\right\|\right], j=-q_{n}+1, \ldots, q_{n}-1$. 
Let $n \geq n_{0}$. If $j \in\left\{-q_{n}+1, \ldots, q_{n}-1\right\}$ and $j^{\prime} \in\left\{-q_{n+1}+1, \ldots, q_{n+1}-1\right\}$ are distinct, then the intervals $I_{n}^{j}$ and $I_{n+1}^{j^{\prime}}$ are disjoint, since otherwise by $\left\|\left(j^{\prime}-j\right) \alpha\right\| \leq \frac{1}{2}\left\|q_{n+1} \alpha\right\|+$ $\frac{1}{2}\left\|q_{n+2} \alpha\right\|<\left\|q_{n+1} \alpha\right\|$, with $0<\left|j^{\prime}-j\right|<q_{n}+q_{n+1} \leq q_{n+2}$ which contradicts (5).

If $\inf _{0 \leq|j|<q_{n}}\|\beta-j \alpha\|<\frac{1}{2}\left\|q_{n+1} \alpha\right\|$ for $n \geq n_{0}$, then there is a sequence $\left(j_{n}\right)_{n \geq n_{0}}$ such that $0 \leq\left|j_{n}\right|<q_{n}$ and $\beta \in I_{n}^{j_{n}}$ for $n \geq n_{0}$.

Since $\beta \in I_{n}^{j_{n}} \cap I_{n+1}^{j_{n+1}}$, we have $j_{0}:=j_{n_{0}}=j_{n_{1}}=\ldots$ This implies $\beta=\left\{j_{0} \alpha\right\}$ which completes the proof of 1 ).

2a) By part 1) if $\beta \notin \mathbb{Z} \alpha+\mathbb{Z}$, it follows that there exists a sequence $\left(n_{k}\right)$ such that $\|\beta-j \alpha\| \geq \frac{1}{2}\left\|q_{n_{k}+1} \alpha\right\|$, for $0 \leq|j| \leq q_{n_{k}}$ and $k \geq 1$. Suppose additionally that $\alpha$ is of bounded type. Since $\left\|q_{n_{k}+1} \alpha\right\|$ and $\frac{1}{q_{n_{k}}}$ are comparable, there is $c>0$ such that $\|\beta-j \alpha\| \geq c / q_{n_{k}}$ for $0 \leq|j| \leq q_{n_{k}}$.

2b) Now let $\beta=\frac{t}{r} \alpha+\frac{u}{s} \notin \mathbb{Z} \alpha+\mathbb{Z}$ with $t, r, u, s$ integers and $r, s \geq 1$. Let $j_{n}$ be such that $\varepsilon_{n}:=\min _{j: 0 \leq|j| \leq q_{n}}\left\|\frac{t}{r} \alpha+\frac{u}{s}-j \alpha\right\|=\left\|\frac{t}{r} \alpha+\frac{u}{s}-j_{n} \alpha\right\|>0$.

We have $\frac{t}{r} \alpha+\frac{u}{s}=j_{n} \alpha+\ell_{n} \pm \varepsilon_{n}$, for an integer $\ell_{n}$; hence: $\left(r s j_{n}-t s\right) \alpha=r u-r s \ell_{n} \pm r s \varepsilon_{n}$. It follows

$$
\left\|\left(r s j_{n}-t s\right) \alpha\right\| \leq r s\left|\varepsilon_{n}\right|
$$

Suppose that $r s j_{n}-t s=0$ for infinitely many $n$. Then $\frac{t}{r}=j_{n}$ and $\left|u-s \ell_{n}\right|=s\left|\varepsilon_{n}\right|$. Since $\left|\varepsilon_{n}\right|$ is arbitrarily small for $n$ large enough and $u, s, \ell_{n}$ are integers, it follows $u=s \ell_{n}$. Then, we find $\beta=j_{n} \alpha+\ell_{n}$ contrary to the assumption that $\beta$ is not in $\mathbb{Z} \alpha+\mathbb{Z}$. It follows that the integers $r s j_{n}-t s$ are different from zero for all $n \geq n_{1}$.

Now, $\alpha$ is of bounded type, so there is $K>0$ such that $q_{n+r s+1} \leq K q_{n}$, for every $n \geq 1$. Using additionally (41) and (5), we obtain

$$
\frac{1}{2 K q_{n}} \leq \frac{1}{2 q_{n+r s+1}} \leq\left\|q_{n+r s} \alpha\right\| \leq\|k \alpha\|, \text { for } 1 \leq|k|<q_{n+r s+1} .
$$

On the other hand, in view of (2), given any constant $C>0$ we have

$$
q_{n+m} \geq m q_{n}+C
$$

for all $m \geq 1$ and $n$ large enough (indeed, it suffices to consider $n$ so that $q_{n-1} \geq C$ ). Hence, for the integer $\left|r s j_{n}-t s\right|$ we have

$$
0<\left|r s j_{n}-t s\right| \leq r s q_{n}+|t| s \leq q_{n+r s+1}
$$

whenever $n$ is large enough. Therefore, for $n$ large enough, by (7) and (8), we obtain

$$
\left|\varepsilon_{n}\right| \geq c / q_{n}, \text { with } c=\frac{1}{2 K}
$$

By taking $c>0$ smaller if necessary, the conclusion holds for all $n \geq 1$. 
Lemma 2.3. Suppose $\alpha$ of bounded type. Let $B$ be a non-empty finite subset of $(\mathbb{Q} \beta+$ $\mathbb{Q} \alpha+\mathbb{Q})-(\mathbb{Z} \alpha+\mathbb{Z})$, where $\beta$ is a real number. Then there exist $c>0$ and a strictly increasing sequence $\left(n_{k}\right)$ such that

$$
\forall \beta_{i} \in B, \forall k \geq 1,\left\|\beta_{i}-j \alpha\right\| \geq c / q_{n_{k}}, \text { for } 0 \leq|j| \leq q_{n_{k}} .
$$

Proof. We have $B=B_{0} \cup B_{1}$, where the elements $\beta_{i}$ in $B_{0}$ are of the form $\beta_{i}=\frac{u_{i}}{s_{i}} \alpha+\frac{w_{i}}{s_{i}}$, with $u_{i}, w_{i}, s_{i}$ integers, $s_{i} \neq 0, \beta_{i} \notin \mathbb{Z} \alpha+\mathbb{Z}$, and the elements in $B_{1}$ of the form $\beta_{i}=$ $\frac{v_{i}}{s_{i}} \beta+\frac{u_{i}}{s_{i}} \alpha+\frac{w_{i}}{s_{i}}$, with $v_{i}, u_{i}, w_{i}, s_{i}$ integers and $v_{i}, s_{i} \neq 0$. Remark that $B_{0}$ or $B_{1}$ can be empty and that $B=B_{0}$ if $\beta \in \mathbb{Q} \alpha+\mathbb{Q}$.

If $\beta \notin \mathbb{Q} \alpha+\mathbb{Q}$ and $B_{1}$ is not empty, we apply Lemma 2.2 to $\beta^{\prime}:=\left(\prod v_{\ell}\right) \beta$. Let $M=\left(\max s_{\ell}\right)\left(\prod v_{\ell}\right), M_{i}=s_{i} \prod_{\ell \neq i} v_{\ell}$. We have $\frac{v_{i}}{s_{i}} \beta=\frac{\beta^{\prime}}{M_{i}}$. There are a positive constant $c$ and a sequence $\left(n_{k}\right)$ such that

$$
\left\|\beta^{\prime}-j \alpha\right\| \geq \frac{c}{q_{n_{k}}}, 0 \leq|j|<q_{n_{k}}
$$

Since $L_{i}:=M_{i} \frac{u_{i}}{s_{i}}$ and $M_{i} \frac{w_{i}}{s_{i}}$ are integers, we have for $j$ such that $0 \leq\left|M_{i} j-L_{i}\right|<q_{n_{k}}$ :

$$
\begin{aligned}
M_{i}\left\|\frac{v_{i}}{s_{i}} \beta+\frac{u_{i}}{s_{i}} \alpha+\frac{w_{i}}{s_{i}}-j \alpha\right\| & \geq\left\|M_{i} \frac{v_{i}}{s_{i}} \beta-M_{i}\left(j \alpha-\frac{u_{i}}{s_{i}} \alpha-\frac{w_{i}}{s_{i}}\right)\right\| \\
& =\left\|\beta^{\prime}-\left(M_{i} j-L_{i}\right) \alpha\right\| \geq \frac{c}{q_{n_{k}}} .
\end{aligned}
$$

We have $M_{i}|j|+\left|L_{i}\right| \leq M|j|+L$, with $L:=\max \left|L_{i}\right|$. As $\alpha$ is of bounded type, there are $r$ and $K$ such that $M q_{n-r}+L \leq q_{n} \leq K q_{n-r}$, for all $n \geq 1$. This implies, simultaneously for every $i$ :

$$
\left\|\frac{v_{i}}{s_{i}} \beta+\frac{u_{i}}{s_{i}} \alpha+\frac{w_{i}}{s_{i}}-j \alpha\right\| \geq \frac{1}{M_{i}}\left\|\beta^{\prime}-\left(M_{i} j-L_{i}\right) \alpha\right\| \geq \frac{c}{M K} \frac{1}{q_{n_{k}-r}}, \text { if }|j|<q_{n_{k}-r} .
$$

For $\beta_{i}$ in $B_{0}$, if this subset is non empty, by the part $2 \mathrm{~b}$ ) of the previous lemma any subsequence of $\left(q_{n}\right)$ is "good".

We conclude that the subsequence $\left(q_{n_{k}-r}\right)_{r \geq 1}$ fulfills the assertion of the lemma.

Remark 1. As the proof of Lemma 2.3 shows, the result is true for any change of the part belonging to $\mathbb{Q} \alpha+\mathbb{Q}$ for the elements of $B_{1}$ (that is, we may replace $\frac{u_{i}}{s_{i}} \alpha+\frac{w_{i}}{s_{i}}$, for $i=1, \ldots, t$, by a different element of $\mathbb{Q} \alpha+\mathbb{Q})$. However, each time we change this part, we also change the resulting subsequence $\left(q_{n_{k}}\right)$.

Remark 2. When $\alpha$ is not of bounded type, the set $K(\alpha)=\left\{\beta \in \mathbb{R}: \lim _{n}\left\|q_{n} \beta\right\|=0\right\}$ is an uncountable additive subgroup of $\mathbb{R}$.

Nevertheless, if $\lim _{n}\left\|q_{n} \beta\right\|=0$ and $\beta \notin \mathbb{Z} \alpha+\mathbb{Z}$, the rate of convergence toward 0 is moderate, as shown by the following lemma (see [Co80, [La88, [KrLi91, Co09]).

Lemma 2.4. If there exists $n_{0}$ such that $\left\|q_{n} \beta\right\| \leq \frac{1}{4} q_{n}\left\|q_{n} \alpha\right\|$ for $n \geq n_{0}$, then $\beta \in \mathbb{Z} \alpha+\mathbb{Z}$. In particular, if $\alpha$ is of bounded type and $\beta$ satisfies $\lim _{n}\left\|q_{n} \beta\right\|=0$, then $\beta \in \mathbb{Z} \alpha+\mathbb{Z}$. 


\subsection{Essential values of cocycles taking values in Abelian groups}

In this subsection we recall the definition and general results about essential values of a cocycle (see [Sc77, Aa97]).

Let $(X, \mathcal{B}, \mu)$ be a (non-atomic) standard Borel probability space and $T:(X, \mathcal{B}, \mu) \rightarrow$ $(X, \mathcal{B}, \mu)$ an ergodic automorphism. Such an automorphism is then automatically aperiodic (that is, for each $n \geq 1,\left\{x \in X: T^{n} x=x\right\}$ has measure zero).

Assume that $G$ is an Abelian locally compact second countable (l.c.s.c.) group with the $\sigma$-algebra of its Borel sets $\mathcal{B}(G)$ and a fixed Haar measure $m_{G}$ (we will also write $d g$ instead of $m_{G}$ ). Denote by $\bar{G}=G \cup\{\infty\}$ the one-point compactification of $G$ (when $G$ is non compact).

For a measurable function $\varphi: X \rightarrow G$, we denote by $\left(\varphi_{n}\right)$ the cocycle generated by $\varphi$ :

$$
\varphi_{n}(x)=\sum_{k=0}^{n-1} \varphi\left(T^{k} x\right), n \geq 1
$$

and we extend the formula to all $n \in \mathbb{Z}$ so that, for $n, k \in \mathbb{Z}, \varphi_{n+k}(x)=\varphi_{n}(x)+\varphi_{k}\left(T^{n} x\right)$. For simplicity, the function $\varphi$ itself will be called a cocycle. We say that a cocycle $\varphi: X \rightarrow G$ is ergodic if the transformation $T_{\varphi}:(x, g) \rightarrow(T x, g+\varphi(x))$ is ergodic on $X \times G$ for the measure $\mu \times d g$.

Recurrence of a cocycle

Let \|\| be a norm on $\mathbb{R}^{d}$. The inequality $\left\|\varphi_{n+1}(x)\right\|-\left\|\varphi_{n}(T x)\right\| \mid \leq\|\varphi(x)\|$ implies the $T$-invariance of the set $\left\{x: \lim _{n}\left\|\varphi_{n}(x)\right\|=+\infty\right\}$. Therefore by ergodicity this set has measure 0 or 1 , and we have the following alternative: either for $\mu$-a.e. every $x$, $\lim _{n}\left\|\varphi_{n}(x)\right\|=+\infty$ or for $\mu$-a.e. $x \liminf _{n}\left\|\varphi_{n}(x)\right\|<+\infty$.

Definition 1. A cocycle $\left(\varphi_{n}\right)$ over $(X, \mu, T)$ with values in $G=\mathbb{R}^{d}$ is recurrent if $\liminf _{n}\left\|\varphi_{n}(x)\right\|<\infty$, for a.e. $x \in X$. It is transient if $\lim _{n}\left\|\varphi_{n}(x)\right\|=+\infty$, for a.e. $x \in X$.

It can be shown that recurrence for $\left(\varphi_{n}\right)_{n \geq 0}$ is equivalent to conservativity of $T_{\varphi}$ with respect to $\mu \times d g$ and that it implies $\liminf _{n}\left\|\varphi_{n}(x)\right\|=0$ for a.e. $x$.

It is also equivalent to the following property: for each neighborhood $U \ni 0$ and $A \subset X$ of positive measure there exists $N \in \mathbb{Z} \backslash\{0\}$ such that

$$
\mu\left(A \cap T^{-N} A \cap\left[\varphi_{N} \in U\right]\right)>0 .
$$

Remark 3. In order to give a simple example of a recurrent cocycle recall that an increasing sequence $\left(\ell_{n}\right)$ is called a rigidity sequence for $T$ if, in the strong operator topology, $\lim _{n} T^{\ell_{n}}=I$ where $I$ is the identity mapping. Suppose that $\varphi: X \rightarrow G$ is a cocycle such that $\varphi_{\ell_{n}} \rightarrow 0$ in measure. Then $\varphi$ is recurrent; indeed, in (10), $T^{-q_{n}} A$ is almost equal to $A$ while $\left[\varphi_{q_{n}} \in U\right]$ is almost the whole space $X$.

Remark 4 . For each $d \geq 1$, the cocycle generated by a function $\varphi: \mathbb{T} \rightarrow \mathbb{R}^{d}$ over any irrational rotation is recurrent if the components of $\varphi$ have bounded variation and 
integral 0. Indeed by Denjoy-Koksma inequality ([6) $)$, since $\left(\varphi_{q_{n}}\right)$ is a bounded sequence in $\mathbb{R}^{d}$ the condition $\liminf \operatorname{in}_{n}\left\|\varphi_{n}(x)\right\|<\infty$ holds for every $x$.

This applies in particular to all piecewise affine or step functions considered in this paper.

We always consider recurrent cocycles.

A cocycle $\varphi$ is called a coboundary if $\varphi=f-f \circ T$ for a measurable map $f: X \rightarrow G$. Two cocycles $\varphi, \psi$ are called cohomologous if $\varphi-\psi$ is a coboundary.

Regular cocycles. An obvious obstruction to the ergodicity of a cocycle is that $\varphi$ is cohomologous to a cocycle $\psi$ taking its values in a smaller closed subgroup of $G$. This suggests the following definition:

Definition 2. A cocycle $\varphi$ is regular if it is cohomologous to a cocycle $\psi$ with values in a closed subgroup $H$ of $G$ such that $T_{\psi}:(x, h) \rightarrow(T x, h+\psi(x))$ is ergodic on $X \times H$ for the measure $\mu \times d h$, where $d h$ is the Haar measure on $H$.

So, a regular cocycle is "almost" ergodic (up to reduction by cohomology to a smaller closed subgroup).

One of the main tools for studying ergodicity and regularity of a cocycle is the following notion.

Essential value. An element $g \in \bar{G}$ is called an essential value for a cocycle $\varphi$, if for each open neighborhood $U \ni g$ in $\bar{G}$, for each $A \in \mathcal{B}$ of positive measure, there exists $N \in \mathbb{Z}$ such that $\mu\left(A \cap T^{-N} A \cap\left[\varphi_{N} \in U\right]\right)>0$. We denote the set of essential values by $\overline{\mathcal{E}}(\varphi)$ and we set $\mathcal{E}(\varphi):=\overline{\mathcal{E}}(\varphi) \cap G$.

Note that, if $g \in \mathcal{E}(\varphi)$, we have $\mu\left(A \cap T^{-N} A \cap\left[\varphi_{N} \in U\right]\right)>0$ for infinitely many values of $N \in \mathbb{Z}$. Indeed, because $T$ is ergodic and aperiodic, for each $N \in \mathbb{Z} \backslash\{0\}$ we can find a subset $C \subset A, \mu(C)>0$ such that $T^{j} C \cap C=\emptyset$, for $|j| \leq N, j \neq 0$. Since $g \in \mathcal{E}(\varphi)$, there is $N_{1}$ such that $\mu\left(C \cap T^{-N_{1}} C \cap\left[\varphi_{N_{1}} \in U\right]\right)>0$. The property of $C$ implies $\left|N_{1}\right|>|N|$. Iterating this construction, we obtain an infinite sequence $\left(N_{k}\right)$ such that $\mu\left(A \cap T^{-N_{k}} A \cap\left[\varphi_{N_{k}} \in U\right]\right)>0$.

Remark 5. Cocycles with non-trivial essential values must be recurrent. Indeed, assume that $g \in \mathcal{E}(\varphi) \backslash\{0\}$. We show Property (10). Take $U$ a neighborhood of $0 \in G$. Then find $N \in \mathbb{Z}$ so that there is $B \subset X, \mu(B)>0$ such that

$$
B \subset A, T^{N} B \subset A \text { and } \varphi_{N}(B) \subset g+U .
$$

Apply once more the definition of the essential value, this time to the set $T^{N} B$ to find $C \subset X, \mu(C)>0$ and an integer $M \neq N$ such that

$$
C \subset T^{N} B, T^{M} C \subset T^{N} B \text { and } \varphi_{M}(C) \subset g+U .
$$

Now, for $x \in C \subset A$ we have $T^{M-N} x=T^{-N}\left(T^{M} x\right) \in T^{-N}\left(T^{N} B\right)=B \subset A$. Moreover, since $T^{M-N} x \in B$,

$$
\varphi_{M-N}(x)=\varphi_{M}(x)+\varphi_{-N}\left(T^{M} x\right)=\varphi_{M}(x)-\varphi_{N}\left(T^{M-N} x\right) \in U-U .
$$


It turns out that $\mathcal{E}(\varphi)$ is a closed subgroup of $G$. Besides, two cohomologous cocycles have the same group of essential values.

Let $\sigma_{g}(x, h):=(x, g+h), g \in G$, be the action of $G$ on $X \times G$ by translations on the second coordinate. Clearly, it commutes with $T_{\varphi}$. Then (see [Sc77], Theorem 5.2) $\mathcal{E}(\varphi)$ is the stabilizer of the Mackey action of $\varphi$, that is

$$
\mathcal{E}(\varphi)=\left\{g \in G: F \circ \sigma_{g}=F, \forall \text { measurable } T_{\varphi} \text {-invariant } F: X \times G \rightarrow \mathbb{C}\right\} .
$$

In other words $\mathcal{E}(\varphi)$ is the group of periods of the measurable $T_{\varphi}$ - invariant functions. Therefore $\varphi$ is ergodic if and only if $\mathcal{E}(\varphi)=G$. If $\varphi$ is regular, then the group $H$ in the definition of regularity is necessarily $\mathcal{E}(\varphi)$. Coboundaries are precisely regular cocycles $\varphi$ with $\mathcal{E}(\varphi)=\{0\}$.

The following lemmas show how essential values and regularity behave when a group homomorphism is applied to a cocycle.

Lemma 2.5. Assume that $\varphi: X \rightarrow G$ is a cocycle and let $M: G \rightarrow H$ be a (continuous) group homomorphism. Then $M \mathcal{E}(\varphi) \subset \mathcal{E}(M \varphi)$. If $M$ is an isomorphism, then $M \mathcal{E}(\varphi)=$ $\mathcal{E}(M \varphi)$.

Proof. Let $p \in \mathcal{E}(\varphi)$. We want to show that $M p$ is a period of the measurable $T_{M \varphi^{-}}$ invariant functions on $X \times H$. Let $F: X \times H \rightarrow \mathbb{C}$ be such a function. Moreover, by a standard argument, we can modify $F$ on a set of zero measure in order to obtain a function (still denoted by $F$ ) which is $T_{M \varphi}$-invariant everywhere.

Let us fix $h \in H$ and denote $F_{h}: X \times G \rightarrow \mathbb{C}$ by setting $F_{h}(x, y)=F(x, h+M y)$. We have

$$
\begin{aligned}
\left(F_{h} \circ T_{\varphi}\right)(x, y) & =F_{h}(T x, y+\varphi(x))=F(T x, h+M y+M \varphi(x)) \\
& =F(x, h+M y)=F_{h}(x, y) .
\end{aligned}
$$

In view of (11), $p \in \mathcal{E}(\varphi)$ is a period for $F_{h}$, i.e., $F_{h}(x, y+p)=F_{h}(x, y)$ for a.e. $(x, y)$. This implies that, for every $h \in H$ and for a.e. $(x, y), F(x, h+M y+M p)=F(x, h+M y)$.

By Fubini, this implies that there is $y \in G$ such that for a.e. $(x, h), F(x, h+M y+M p)=$ $F(x, h+M y)$. By invariance of the Haar measure, this implies $F(x, h+M p)=F(x, h)$, for a.e. $(x, y)$ and $M p$ is a period of $F$.

For the second part of the assertion, apply the above to $M \varphi$ and $M^{-1}$.

We have the following lemma (cf. Lemma 2.9 in [CoFr11]):

Lemma 2.6. If $\varphi$ is a cocycle on $(X, \mu, T)$ with values in an Abelian l.c.s.c. group $G$ and $H$ a closed subgroup of $G$, then the subgroup $\mathcal{E}(\varphi) / H$ of $G / H$ is such that

$$
\mathcal{E}(\varphi) / H \subset \mathcal{E}(\varphi+H)
$$

If $H \subset \mathcal{E}(\varphi)$, then we have

$$
\mathcal{E}(\varphi+H)=\mathcal{E}(\varphi) / H
$$

Moreover, $\varphi^{*}:=\varphi+H: X \rightarrow G / H$ is regular if and only if $\varphi$ is regular. 
Proof. Whenever $H \subset G$ is a closed subgroup, (12) follows from Lemma 2.5 applied to the homomorphism $g \in G \rightarrow g+H \in G / H$.

Now suppose that $H \subset \mathcal{E}(\varphi)$. In view of (12) it remains to show that $\mathcal{E}(\varphi+H) \subset \mathcal{E}(\varphi) / H$. Take $g_{0}+H \in \mathcal{E}(\varphi+H)$. All we need to show is that there exists $h_{0} \in H$ such that $g_{0}+h_{0} \in \mathcal{E}(\varphi)$, which, by $H \subset \mathcal{E}(\varphi)$, is equivalent to showing that $g_{0} \in \mathcal{E}(\varphi)$.

Take $F: X \times G \rightarrow \mathbb{C}$ which is measurable and $T_{\varphi^{-}}$invariant. Since $H \subset \mathcal{E}(\varphi), F \circ \sigma_{h}=F$ for each $h \in H$ because of (11). We can defined $\tilde{F}$ on $X \times G / H$ such that $\tilde{F}(x, g+H)=$ $F(x, g)$. Since $g_{0}+H \in \mathcal{E}(\varphi+H)$, again using (11), we obtain that $\tilde{F} \circ \sigma_{g_{0}+H}=\tilde{F}$, which by $H$-invariance of $F$ means $F \circ \sigma_{g_{0}}=F$ and therefore $g_{0} \in \mathcal{E}(\varphi)$.

Assume now that $\varphi^{*}$ is regular. So there are a measurable $\eta^{*}: X \rightarrow G / H$ and a closed subgroup $J^{*} \subset G / H$ such that

$$
\psi^{*}(x):=\varphi^{*}(x)+\eta^{*}(x)-\eta^{*}(T x) \in J^{*} \subset G / H
$$

and $T_{\psi^{*}}$ is ergodic on $X \times J^{*}$, i.e. $\mathcal{E}\left(\psi^{*}\right)=J^{*}$. Let $\pi: G \rightarrow G / H$ be the canonical homomorphism and $s: G / H \rightarrow G$ a measurable selector, that is, $s(g+H) \in g+H$ for each $g+H \in G / H$. Then $J:=\pi^{-1}\left(J^{*}\right)$ is a closed subgroup of $G$. Denote $\eta:=s \circ \eta^{*}$ and set

$$
\varphi^{\prime}(x):=\varphi(x)+\eta(x)-\eta(T x) .
$$

Then $\varphi^{\prime}(x)+H=\varphi^{*}(x)+\eta^{*}(x)-\eta^{*}(T x)=\psi^{*}(x) \in J^{*}$, whence $\varphi^{\prime}: X \rightarrow J$. By (13), since $\mathcal{E}\left(\varphi^{\prime}\right)=\mathcal{E}(\varphi)$, we have

$$
\mathcal{E}\left(\varphi^{\prime}\right) / H=\mathcal{E}(\varphi) / H=\mathcal{E}(\varphi+H)=\mathcal{E}\left(\varphi^{*}\right)=J^{*},
$$

so $\mathcal{E}\left(\varphi^{\prime}\right)=J$ and $\varphi$ is regular.

Conversely, if $\varphi$ is regular then $\varphi=\eta-\eta \circ T+\psi$, where $\eta: X \rightarrow G$ is measurable and $\psi: X \rightarrow \mathcal{E}(\varphi)$. Then $\varphi^{*}$ is cohomologous to $\psi+H$ which takes values in $\mathcal{E}(\psi) / H=$ $\mathcal{E}(\varphi) / H=\mathcal{E}(\varphi+H)$ by $(13)$, so $\varphi^{*}$ is regular.

A particular case is when $H=\mathcal{E}(\varphi)$. For $\varphi^{*}=\varphi+\mathcal{E}(\varphi)$, we get: $\mathcal{E}\left(\varphi^{*}\right)=\{0\}$ and $\varphi$ is regular if and only if $\varphi^{*}$ is regular (hence a coboundary).

It can be shown that a cocycle $\varphi$ is a coboundary if and only if $\overline{\mathcal{E}}(\varphi)=\{0\}$. This includes in particular the fact that, if $\varphi$ has its values in a compact group and has no non trivial essential values, it is a coboundary.

Hence regularity is equivalent to $\overline{\mathcal{E}}\left(\varphi^{*}\right)=\{0\}$. In particular cocycles with values in compact groups, or more generally such that $\mathcal{E}(\varphi)$ has a compact quotient in $G$, are regular.

Lemma 2.7. Assume that $\varphi: X \rightarrow G$ is a cocycle and let $M: G \rightarrow H$ be a (continuous) group homomorphism. If $\varphi: X \rightarrow G$ is regular, so is $M \varphi: X \rightarrow H$.

Proof. If $\varphi$ is regular, there is a cocycle $\psi: X \rightarrow J$ with values in a closed subgroup $J \subset G$ and a measurable function $f: X \rightarrow G$ such that $\varphi=f-f \circ T+\psi$ and $T_{\psi}:(x, j) \rightarrow(T x, j+\psi(x))$ is ergodic on $X \times J$. Thus $M \varphi=M f-(M f) \circ T+M \psi$. 
We have $\mathcal{E}(\psi)=J$ by ergodicity of $T_{\psi}$ on $X \times J$ and $M J=M \mathcal{E}(\psi) \subset \mathcal{E}(M \psi)$ by Lemma 2.5. Since $M \psi: X \rightarrow M J \subset \overline{M J}$, it implies $\mathcal{E}(M \psi) \subset \overline{M J}$. But $\mathcal{E}(M \psi)$ includes $M J$ and is closed, so it is equal to $\overline{M J}$.

Hence $T_{M \psi}$ is ergodic on $X \times \overline{M J}$, which implies the regularity of $M \varphi$.

The lemma gives a variant of the proof of the second part of Lemma 2.6. It shows that if $\varphi$ has a non regular quotient then it is non regular.

Remark 6. Assume that $\psi: X \rightarrow G_{1} \times G_{2}$ is a cocycle of the form $\psi=\left(0, \psi_{2}\right)$ with $\psi_{2}: X \rightarrow G_{2}$. Then $\mathcal{E}(\psi)=\{0\} \times \mathcal{E}\left(\psi_{2}\right)$. Indeed, $\psi_{N}(x)$ is close to $\left(g_{1}, g_{2}\right)$ if and only if $g_{1}$ is close to zero and $\left(\psi_{2}\right)_{N}(x)$ is close to $g_{2}$, so this equality follows directly from the definition of essential value. Moreover, clearly $\psi$ is a regular cocycle if $\psi_{2}$ is regular and the converse follows from Lemma 2.7.

Finally we recall some effective tools which can be used to find essential values of a cocycle. Given $T:(X, \mathcal{B}, \mu) \rightarrow(X, \mathcal{B}, \mu)$ and $\varphi: X \rightarrow G$, we denote the image of $\mu$ on $G$ via $\varphi$ by $\varphi_{*} \mu$. We will make use of the following essential value criterion.

Proposition 2.8 ([LePaVo96]). Assume that $T$ is ergodic and let $\varphi: X \rightarrow G$ be a cocycle with values in an Abelian l.c.s.c. group $G$. Let $\left(\ell_{n}\right)$ be a rigidity sequence for $T$. If $\left(\varphi_{\ell_{n}}\right)_{*} \mu \rightarrow \nu$ weakly on $\bar{G}$, then $\operatorname{supp}(\nu) \subset \overline{\mathcal{E}}(\varphi)$.

Let us recall that all Abelian l.c.s.c. groups are metrizable. Let $d$ be a metric.

Definition 3. We say that $g \in G$ is a quasi-period of a cocycle $\varphi$ over $T$ with values in $G$, if there exist $\delta>0$, a rigidity sequence $\left(\ell_{n}\right)$ for $T$ and a sequence $0<\varepsilon_{n} \rightarrow 0$ such that

$$
\mu\left(A_{n}\right) \geq \delta, \forall n \geq 1, \text { where } A_{n}=\left\{x \in X: d\left(\varphi_{\ell_{n}}(x), g\right)<\varepsilon_{n}\right\} .
$$

Lemma 2.9. The set of quasi-periods is included in $\mathcal{E}(\varphi)$.

Proof. With no loss of generality we can assume that $\left(\varphi_{\ell_{n}}\right)_{*} \mu \rightarrow \nu$ where $\nu$ is a probability measure on $\bar{G}$. In view of Proposition 2.8 it suffices to show that a quasi-period $g$ is in the topological support of $\nu$. Take $U$ a neighborhood of $g$, and select a smaller neighborhood $g \in V \subset U$ so that $\bar{V} \subset U$. We have $\nu(U) \geq \lim \sup \left(\varphi_{\ell_{n}}\right)_{*}(\mu)(V)=$ $\lim \sup \mu\left(\varphi_{\ell_{n}}^{-1}(V)\right) \geq \lim \sup \mu\left(A_{n}\right) \geq \delta$.

The following "lifting essential values" lemma can be applied when $T$ is an irrational rotation by $\alpha, \varphi$ below is $\mathbb{R}$-valued, centered and of bounded variation (see (6) ), dealing with different subsequences of the sequence $\left(q_{n}\right)$ of denominators of $\alpha$.

Lemma 2.10. Assume that $T$ is ergodic and let $\left(\ell_{n}\right)$ be a rigidity sequence of $T$. Assume that $\varphi: X \rightarrow H$ is a cocycle such that there exists a compact neighborhood $C \subset H$ of $0 \in H$ for which $\varphi_{\ell_{n}} \in C$ eventually. Let $\psi: X \rightarrow G$ be a cocycle such that $\left(\psi_{\ell_{n}}\right)_{*}(\mu) \rightarrow \kappa$ with $\kappa$ a probability measure on $\bar{G}$. Assume that

$$
0 \neq g_{0} \in \operatorname{supp}(\kappa) \cap G .
$$

Then there exists $h_{0} \in H$ such that $\left(h_{0}, g_{0}\right) \in \mathcal{E}(\Phi)$, where $\Phi:=(\varphi, \psi): X \rightarrow H \times G$. 
Proof. Note first that in view of Proposition 2.8, $g_{0} \in \mathcal{E}(\psi)$. By passing to a subsequence if necessary, we can assume that the distributions of $\varphi_{\ell_{n}}$ and $\Phi_{\ell_{n}}$ converge, that is

$$
\left(\varphi_{\ell_{n}}\right)_{*}(\mu) \rightarrow \nu, \quad\left(\Phi_{\ell_{n}}\right)_{*}(\mu) \rightarrow \rho,
$$

where $\nu$ is a probability measure on $\bar{H}$, but in fact (by our standing assumption) which is concentrated on $C$, whence $\rho$ is a probability measure concentrated on $C \times \bar{G}$. Moreover,

$$
\text { the projections of } \rho \text { on } C \text { and } \bar{G} \text { are equal to } \nu \text { and } \kappa \text { respectively. }
$$

Using (14), for each $n \geq 1$ select an open neighborhood $G \supset V_{n} \ni g_{0}$ so that $\bar{V}_{n}$ is compact, $\operatorname{diam} \bar{V}_{n}<1 / n, \kappa\left(V_{n}\right)>0$ and $V_{n+1} \subset V_{n}$. In view of (15), $\rho\left(C \times \bar{V}_{n}\right)>0$. Since $C \times \bar{V}_{n}$ is compact, there is $\left(c_{n}, g_{n}\right) \in C \times \bar{V}_{n}$ such that $\left(c_{n}, g_{n}\right) \in \operatorname{supp}(\rho)$ (if no such a point exists, each point of $C \times \bar{V}_{n}$ has a neighborhood which is of measure $\rho$ zero, a finite union of such neighborhoods must then cover the set $C \times \bar{V}_{n}$, a contradiction).

In this way we obtain a sequence $\left(c_{n}, g_{n}\right), n \geq 1$, of points which are in $\operatorname{supp}(\rho) \cap C \times \bar{V}_{1}$ and from which we can choose a converging subsequence $\left(c_{n_{k}}, g_{n_{k}}\right)$. Moreover, by our assumption on the diameters of $V_{n},\left(c_{n_{k}}, g_{n_{k}}\right) \rightarrow\left(c, g_{0}\right)$, so the result follows.

In particular, by the proof of Lemma 2.9, Lemma 2.10 will apply when $g_{0} \in G$ is an essential value of $\psi$ obtained as a quasi-period along a subsequence of the sequence $\left(q_{n}\right)$ of denominators of $\alpha$.

\subsection{Essential values of cocycles taking values in $\mathbb{R}^{d}$}

In the lemmas of this subsection, $\Phi$ will stand for a cocycle with values in $\mathbb{R}^{d}$.

Lemma 2.11. Let $\theta=\left(\theta_{1}, \ldots, \theta_{d}\right) \in \mathbb{R}^{d}$ be a non zero essential value of $\Phi$. Then there is a change of basis in $\mathbb{R}^{d}$ given by a matrix $M$ such that the vector $(1,0, \ldots, 0)$ is an essential value of the cocycle $M \Phi$. If $\theta$ is rational, then $M$ can be taken rational.

Proof. There is a change of basis in $\mathbb{R}^{d}$ with $\theta$ as the first vector of the new basis. This can be done via a matrix $M_{1}$ with rational coefficients if $\theta \in \mathbb{Z}^{d}$. The cocycle $\Phi^{\prime}=M_{1} \Phi$ has an essential value of the form $\left(\theta_{1}, 0, \ldots, 0\right)$, where $\theta_{1}$ is a positive real (a positive integer if $\theta$ is in $\mathbb{Z}^{d}$, for an adapted choice of $M_{1}$ ). By applying a linear isomorphism $M_{2}$ (rational in the $\theta$ rational case) we get that $\Phi^{\prime \prime}=M_{2} M_{1} \Phi$ has an essential value of the form $(1,0, \ldots, 0)$.

Lemma 2.12. There exist a linear isomorphism $M: \mathbb{R}^{d} \rightarrow \mathbb{R}^{d}$ and integers $d_{0}, d_{1}, d_{2} \geq 0$ such that if we set $H_{i}=\mathbb{R}^{d_{i}}, i=0,1,2$, then

$$
\mathbb{R}^{d}=H_{0} \times H_{1} \times H_{2}, \quad M \Phi=\left(\psi_{0}, \psi_{1}, \psi_{2}\right)
$$

with $\psi_{i}: X \rightarrow H_{i}, i=0,1,2$, and $\mathcal{E}(M \Phi)=\{0\} \times H_{1} \times \Gamma_{2}$, with $\Gamma_{2}$ a discrete subgroup of $\mathrm{H}_{2}$ such that $\mathrm{H}_{2} / \Gamma_{2}$ is compact. If $\Phi$ is a coboundary, then $d_{1}=d_{2}=0$ 
Proof. The group $\mathcal{E}(\Phi)$ is a closed subgroup of $\mathbb{R}^{d}$, hence there are linearly independent vectors $v_{1}, \ldots, v_{d_{1}}, w_{1}, \ldots, w_{d_{2}}$ in $\mathbb{R}^{d}$ such that

$$
\mathcal{E}(\Phi)=\left\{s_{1} v_{1}+\ldots+s_{d_{1}} v_{d_{1}}+t_{1} w_{1}+\ldots+t_{d_{2}} w_{d_{2}}: s_{j} \in \mathbb{R}, t_{k} \in \mathbb{Z}\right\} .
$$

Select $y_{1}, \ldots, y_{d_{0}} \in \mathbb{R}^{d}$ so that together with previously chosen $v_{j}$ and $w_{k}$ we obtain a basis of $\mathbb{R}^{d}$. Then define a linear isomorphism $M$ of $\mathbb{R}^{d}$ by setting

$$
M\left(y_{i}\right)=e_{i}, M\left(v_{j}\right)=e_{d_{0}+j}, M\left(w_{k}\right)=e_{d_{0}+d_{1}+k},
$$

where $e_{1}, \ldots, e_{d}$ is the standard basis of $\mathbb{R}^{d}$. Since $\mathcal{E}(M \Phi)=M \mathcal{E}(\Phi)$, we obtain $\mathcal{E}(M \Phi)=$ $\{0\} \times H_{1} \times \Gamma_{2}$ as required and $M \Phi=\left(\psi_{0}, \psi_{1}, \psi_{2}\right)$.

Corollary 2.13. Let us consider the case $d=2$. Let $\Phi=\left(\varphi^{1}, \varphi^{2}\right): X \rightarrow \mathbb{R}^{2}$ be a cocycle such that $\mathcal{E}(\Phi) \neq\{0\}$. Then

$$
\Phi \text { is regular if and only if } a \varphi^{1}+b \varphi^{2}: X \rightarrow \mathbb{R} \text { is regular for each } a, b \in \mathbb{R} \text {. }
$$

Proof. In view of Lemma 2.7 we only need to prove sufficiency. Suppose $\Phi$ is not regular. In view of Lemma 2.12 we obtain a linear isomorphism $M: \mathbb{R}^{2} \rightarrow \mathbb{R}^{2}$ such that $M \Phi=$ $\left(\psi^{0}, \psi^{i}\right)$ with $\psi^{0}: X \rightarrow H_{0}, \psi^{i}: X \rightarrow H_{i}, i$ equals either 1 or 2 and $H_{0} \neq\{0\}$ by non-regularity of $\Phi$ and $H_{i} \neq\{0\}$ since $\mathcal{E}(\Phi) \neq\{0\}$ by hypothesis. Hence $\mathcal{E}\left(\psi^{0}\right)=\{0\}$ and there are $a$ and $b$ such that $\psi^{0}=a \varphi^{1}+b \varphi^{2}$. But $a \varphi^{1}+b \varphi^{2}$ is, by assumption, regular, so $\psi^{0}$ must be a coboundary. Hence $\left(\psi^{0}, \psi^{i}\right)$ is cohomologous to $\left(0, \psi^{i}\right)$ and it now follows from Remark 6 that $\left(\psi^{0}, \psi^{i}\right)$ is regular, a contradiction.

Lemma 2.14. Let $\Phi: X \rightarrow \mathbb{R}^{d}$ be a recurrent cocycle and let $M: \mathbb{R}^{d} \rightarrow \mathbb{R}^{d}$ be a linear isomorphism of $\mathbb{R}^{d}$ yielding the assertions of the previous lemma. Assume additionally that the quotient cocycle $\Phi / \mathcal{E}(\Phi)$ is constant. Then $\psi_{0}=0$. Moreover, $\Phi$ is regular.

Proof. Since $\mathcal{E}(M \Phi)=M \mathcal{E}(\Phi)=\{0\} \times H_{1} \times \Gamma_{2}$, we have

$$
\left(\psi_{0}(x), \psi_{1}(x), \psi_{2}(x)\right) /\{0\} \times H_{1} \times \Gamma_{2}=\text { const } .
$$

It follows that there is a constant $b \in \mathbb{R}^{d_{0}}$ such that $\psi_{0}=b$. However, $M \Phi$ is recurrent as $\Phi$ is recurrent and therefore $\psi_{0}$ is also recurrent. It follows that $b=0$. Now regularity follows from Remark [6] since $H_{1} \times \Gamma_{2}$ has a compact quotient in $H_{1} \times H_{2}$.

An example of a situation described by the previous lemma is the following: let $\psi$ be an ergodic step cocycle with values in $\mathbb{Z}$ over an irrational rotation by $\alpha \in(0,1)$. If we modify $\psi$ by $1_{[0, \alpha)}-\alpha$ which is a coboundary, then for $\varphi:=\psi+1_{[0, \alpha)}-\alpha$ we have $\mathcal{E}(\varphi)=\mathcal{E}(\psi)=\mathbb{Z} ;$ here $\Gamma_{2}=\mathbb{Z}$ and $\varphi \bmod \mathcal{E}(\varphi)=-\alpha$. 


\section{Step cocycles over an irrational rotation}

In this section, we study the regularity of a step $\mathbb{R}^{d}$-valued cocycle $\Phi=\left(\varphi^{1}, \ldots, \varphi^{d}\right)$ over an irrational rotation $T: x \rightarrow x+\alpha$. For such a cocycle the coordinate $\mathbb{R}$-valued cocycles $\varphi^{j}$ are integrable and we will constantly assume that $\int_{0}^{1} \varphi^{j} d \mu=0$ with $\mu=m_{\mathbb{T}}$ the Lebesgue measure on $\mathbb{T}^{1}$, for $j=1, \ldots, d$.

Representations of step cocycles

The coordinates of $\Phi=\left(\varphi^{1}, \ldots, \varphi^{d}\right)$ can be (uniquely) represented as follows:

$$
\varphi^{j}(x)=\sum_{i} t_{i, j}\left(1_{I_{i, j}}(x)-\mu\left(I_{i, j}\right)\right),
$$

where, for $j=1, \ldots, d,\left\{I_{i, j}\right\}$ is a finite family of disjoint intervals of $[0,1)$ (covering $[0,1)$ and maximal on which $\varphi^{j}$ is constant) and $t_{i, j} \in \mathbb{R}$. Clearly, when $d \geq 1$ is fixed, the family of step cocycles forms a linear space over $\mathbb{R}$.

Setting $\beta_{i, j}=\mu\left(I_{i, j}\right)$ and $\psi^{i, j}=1_{I_{i, j}}-\beta_{i, j}$, we have $\psi_{n}^{i, j}(x)=\sum_{k=0}^{n-1} 1_{I_{i, j}}(x+k \alpha)-n \beta_{i, j}$; hence the cocycle $\varphi_{n}^{j}$ can be written in the following form:

$$
\varphi_{n}^{j}(x)=\sum_{i} t_{i, j} \psi_{n}^{i, j}(x)=\sum_{i} t_{i, j}\left(u_{(n)}^{i, j}(x)-\left\{n \beta_{i, j}\right\}\right),
$$

with the notation (which is not a cocycle expression)

$$
u_{(n)}^{i, j}(x):=\psi_{n}^{i, j}(x)+\left\{n \beta_{i, j}\right\}=\sum_{k=0}^{n-1} 1_{I_{i, j}}(x+k \alpha)-\left[n \beta_{i, j}\right] \in \mathbb{Z} .
$$

Remark 7. Without loss of generality, we can assume that the difference between any two discontinuity points of the cocycle $\Phi$ is never a multiple of $\alpha$ (modulo 1). Indeed, if $\beta$ and $\beta^{\prime}$ are two discontinuity points of a component of $\Phi$ such that $\beta^{\prime}-\beta \in \mathbb{Z} \alpha+\mathbb{Z}$, we can suppress one of them by adding to $\Phi$ a coboundary, without changing the ergodic properties of $\Phi$ (we use the fact that $1_{\left[\beta, \beta^{\prime}\right)}(x)-\left(\beta^{\prime}-\beta\right)$ is coboundary 1 ). In particular, after modification, the lengths $\mu\left(I_{i, j}\right)$ in the representation of the new ccocycle are not in $\mathbb{Z} \alpha+\mathbb{Z}$.

Rational step cocycles Assume that $\varphi: \mathbb{T} \rightarrow \mathbb{R}$ is a zero mean step cocycle with its unique representation (17) of the form

$$
\varphi=\sum_{i=1}^{m} t_{i}\left(1_{I_{i}}-\mu\left(I_{i}\right)\right) .
$$

\footnotetext{
${ }^{1}$ Indeed, we have $1_{[1-\alpha, 1)}(x)-\alpha=j(x)-j(x+\alpha)$ with $j(x)=\{x\}$, then for integers $k, s$

$$
1_{[1-\{k \alpha+s\}, 1)}(x)-\{k \alpha+s\}=1_{[1-\{k \alpha\}, 1)}(x)-\{k \alpha\}=j(x)-j(x+k \alpha)=j_{k}(x)-j_{k}(x+\alpha) .
$$
}

The general case is obtained using the obvious fact that other rotations commute with $T x=x+\alpha$. 
We say that $\varphi$ is rational if there are $c_{i} \in \mathbb{Q}, i=1, \ldots, m$ and $\beta \in \mathbb{R}$ such that

$$
\varphi=\sum_{i=1}^{m} c_{i} 1_{I_{i}}-\beta
$$

Lemma 3.1. Assume that $\varphi: \mathbb{T} \rightarrow \mathbb{R}$ is a (zero-mean) step cocycle. The following conditions are equivalent:

(i) $\varphi$ is rational.

(ii) There exists $w \in \mathbb{R}$ such that in the unique representation (20) of $\varphi$ we have $t_{i} \in w+\mathbb{Q}$ for $i=1, \ldots, m$.

(iii) $\varphi$ takes values in a coset of $\mathbb{Q}$.

In particular, the family of rational cocycles is a linear space over $\mathbb{Q}$.

Proof. (i) $\Rightarrow\left(\right.$ ii) By (20),$\varphi=\sum_{i=1}^{m} t_{i} 1_{I_{i}}-\gamma$, where $\gamma=\sum_{i=1}^{m} t_{i} \mu\left(I_{i}\right)$. For $x \in I_{i}$ we have

$$
c_{i}-\beta=\varphi(x)=t_{i}-\gamma,
$$

so $t_{i} \in(\gamma-\beta)+\mathbb{Q}$ for $i=1, \ldots, m$.

(ii) $\Rightarrow$ (iii) For some $r_{i} \in \mathbb{Q}, i=1, \ldots, m$ and $x \in[0,1)$ we have

$$
\varphi(x)=\sum_{i=1}^{m}\left(w+r_{i}\right)\left(1_{I_{i}}(x)-\mu\left(I_{i}\right)\right)=\sum_{i=1}^{m} r_{i} 1_{I_{i}}(x)+(w-\gamma) \in(w-\gamma)+\mathbb{Q} .
$$

(iii) $\Rightarrow$ (i) Take the unique representation (20) of $\varphi: \varphi=\sum_{i=1}^{m} t_{i} 1_{I_{i}}-\gamma$ with $\gamma=$ $\sum_{i=1}^{m} t_{i} \mu\left(I_{i}\right)$. By assumption, there exists $\eta \in \mathbb{R}$ such that $\varphi(x) \in \eta+\mathbb{Q}$ for each $x \in[0,1)$. Thus, for $x \in I_{i}$ we have

$$
t_{i}-\gamma=\varphi(x)=\eta+r_{i}
$$

for some $r_{i} \in \mathbb{Q}$. Whence $t_{i} \in(\gamma+\eta)+\mathbb{Q}$ for $i=1, \ldots, m$.

The latter assertion follows directly from (iii).

Suppose that $\varphi$ is rational with a representation (21) and let $\varphi=\sum_{i=1}^{m} c_{i}^{\prime} 1_{I_{i}}-\beta^{\prime}$ (with $c_{i}^{\prime} \in \mathbb{Q}$ ) be another rational representation. Then by (iii) of Lemma 3.1 it follows that $\beta-\beta^{\prime} \in \mathbb{Q}$, in other words, in the rational representation (21) the $\operatorname{coset} \beta+\mathbb{Q} \in \mathbb{R} / \mathbb{Q}$ is unique. By $\beta(\varphi)$ we will denote that coset (in fact, less formally it will be the number $\beta$ in $(21)$ understood modulo $\mathbb{Q}$ ). Note that

$$
\varphi(x) \in \beta(\varphi) \text { for all } x \in \mathbb{T} \text {. }
$$

With this in mind we have immediately the following observation:

Lemma 3.2. Assume that $\varphi^{1}, \ldots, \varphi^{d}: \mathbb{T} \rightarrow \mathbb{R}$ are rational step cocycles. Assume moreover that $a_{j} \in \mathbb{Q}$ for $j=1, \ldots, d$ and $\operatorname{set} \varphi=\sum_{j=1}^{d} a_{j} \varphi^{j}$. Then

$$
\beta(\varphi)=\sum_{j=1}^{d} a_{j} \beta\left(\varphi^{j}\right)
$$


Now, let $d \geq 1$. We say that a step cocycle $\Phi: \mathbb{T} \rightarrow \mathbb{R}^{d}$ is a rational step cocycle if its coordinates $\varphi^{j}$ are rational, i.e.:

$$
\varphi^{j}=\sum_{i} c_{i, j} 1_{I_{i, j}}-\beta_{j}
$$

where the coefficients $c_{i, j}$ are rational numbers and $\beta_{j}$ is such that $\int_{0}^{1} \varphi^{j} d \mu=0, j=$ $1, \ldots, d$.

In this case, by replacing $\Phi$ by its non-zero integer multiple so that all $c_{i, j}$ are integers (recall that a non-zero multiple of a cocycle $\Phi$ shares its ergodic properties with $\Phi$ ) we obtain:

$$
\varphi_{n}^{j}(x)=u_{(n)}^{j}(x)-\left\{n \beta_{j}\right\}, n \geq 1
$$

where the functions $u_{(n)}^{j}$ have values in $\mathbb{Z}$.

Below we write $\beta_{j}=\beta_{j}\left(\varphi^{j}\right)=\beta_{j}(\Phi)$ (in the representation (22) ) to stress the dependence of the $\beta_{j}$ 's on the cocycle $\Phi$. The number of discontinuities of $\Phi$ is denoted $D(\Phi)$.

We denote by $\mathcal{L}\left(\beta_{j}\right)$ the set of limit values of the sequence $\left(\left\|q_{n} \beta_{j}\right\|\right)_{n \geq 1}$. Observe that if $\mathcal{L}\left(\beta_{j}\right) \neq\{0\}$, there exists a sequence $\left(n_{k}\right)$ such that $\lim _{k}\left\{q_{n_{k}} \beta_{j}\right\} \in(0,1)$.

Let $L:=\max _{i, j} V\left(\psi^{i, j}\right)$ in case (18), or $L:=\max _{j} V\left(\varphi^{j}\right)$ in case (23), where $V$ is the variation.

$\mathcal{F}$ will denote the interval of integers

$$
\mathcal{F}=\{\ell \in \mathbb{Z}:|\ell| \leq L+1\}
$$

From (ㅁ), (23) and (19), it follows that:

$$
u_{\left(q_{n}\right)}^{j}(x) \in \mathcal{F}, u_{\left(q_{n}\right)}^{i, j}(x) \in \mathcal{F} .
$$

\subsection{Rational step cocycles}

Lemma 3.3. Let $\Phi$ be a rational step cocycle. If $\mathcal{L}\left(\beta_{j_{0}}\right) \neq\{0\}$ for some $j_{0}$, then $\mathcal{E}(\Phi)$ contains a rational vector $\theta=\left(\theta_{1}, \ldots, \theta_{d}\right)$ with $\theta_{j_{0}} \neq 0$.

Proof. By multiplying $\Phi$ by an integer if needed, we can use (23) with $u_{(n)}^{j}(x) \in \mathbb{Z}$. We can select a subsequence $\left(n_{k}\right)$ so that $\left(\left\{q_{n_{k}} \beta_{j}\right\}\right)_{k \geq 1}$ converges for all $j=1, \ldots, d$ to a limit denoted $\delta_{j}$, with $\delta_{j_{0}} \in(0,1)$. Taking into account (25), denote for $\left(\ell_{1}, \ldots, \ell_{d}\right) \in \mathcal{F}^{d}$

$$
A_{k, \ell_{1}, \ldots, \ell_{d}}=\left\{x \in \mathbb{T}: u_{\left(q_{n_{k}}\right)}^{j}(x)=\ell_{j}, j=1, \ldots, d\right\} .
$$

Note that, for each $k \geq 1,\left\{A_{k, \ell_{1}, \ldots, \ell_{d}}:\left(\ell_{1}, \ldots, \ell_{d}\right) \in \mathcal{F}^{d}\right\}$ is a partition of $\mathbb{T}$. By passing to a further subsequence if necessary, we can assume that $\mu\left(A_{k, \ell_{1}, \ldots, \ell_{d}}\right) \rightarrow$ 
$\gamma_{\ell_{1}, \ldots, \ell_{d}}$ when $k \rightarrow \infty$, for each $\left(\ell_{1}, \ldots, \ell_{d}\right) \in \mathcal{F}^{d}$. In view of (24), (23) and the fact that $\int \varphi^{j} d \mu=0$, we have

$$
\begin{aligned}
& \sum_{\ell \in \mathcal{F}} \ell \mu\left(\cup_{\ell_{1}, \ldots, \ell_{j_{0}-1}, \ell_{j_{0}+1}, \ldots, \ell_{d} \in \mathcal{F}} A_{k, \ell_{1}, \ldots, \ell_{j_{0}-1}, \ell, \ell_{j_{0}+1}, \ldots, \ell_{d}}\right) \\
= & \int_{0}^{1} u_{\left(q_{n_{k}}\right)}^{j_{0}}(x) d x=\int_{0}^{1}\left(\varphi_{q_{n_{k}}}^{j_{0}}(x)+\left\{q_{n_{k}} \beta_{j_{0}}\right\}\right) d x=\left\{q_{n_{k}} \beta_{j_{0}}\right\} \rightarrow \delta_{j_{0}} .
\end{aligned}
$$

It follows that

$$
\sum_{\ell \in \mathcal{F}} \ell \sum_{\ell_{1}, \ldots, \ell_{j_{0}-1}, \ell_{j_{0}+1}, \ldots, \ell_{d} \in \mathcal{F}} \gamma_{\ell_{1}, \ldots, \ell_{j_{0}-1}, \ell, \ell_{j_{0}+1}, \ldots, \ell_{d}}=\delta_{j_{0}}
$$

with $\delta_{j_{0}} \in(0,1)$. Hence there are $\underline{\ell} \neq \underline{\ell}^{\prime}$ such that

$\sum_{\ell_{1}, \ldots, \ell_{j_{0}-1}, \ell_{j_{0}+1}, \ldots, \ell_{d} \in \mathcal{F}} \gamma_{\ell_{1}, \ldots, \ell_{j_{0}-1}, \underline{\ell}, \ell_{j_{0}+1}, \ldots, \ell_{d}}>0, \sum_{\ell_{1}, \ldots, \ell_{j_{0}-1}, \ell_{j_{0}+1}, \ldots, \ell_{d} \in \mathcal{F}} \gamma_{\ell_{1}, \ldots, \ell_{j_{0}-1}, \underline{\ell}^{\prime}, \ell_{j_{0}+1}, \ldots, \ell_{d}}>0$.

Indeed otherwise, $\sum_{\ell_{1}, \ldots, \ell_{j_{0}-1}, \ell_{j_{0}+1}, \ldots, \ell_{d} \in \mathcal{F}} \gamma_{\ell_{1}, \ldots, \ell_{j_{0}-1}, \ell_{0}, \ell_{j_{0}+1}, \ldots, \ell_{d}}=1$ for some $\ell_{0} \in \mathcal{F}$ and the other sums are 0 , so that the left hand side of $(26)$ is an integer, a contradiction. This implies

$$
\gamma_{\ell_{1}, \ldots, \ell_{j_{0}-1}, \underline{\ell}, \ell_{j_{0}+1}, \ldots, \ell_{d}}>0, \gamma_{\ell_{1}^{\prime}, \ldots, \ell_{j_{0}-1}^{\prime}, \underline{\ell}^{\prime}, \ell_{j_{0}+1}^{\prime}, \ldots, \ell_{d}^{\prime}}>0
$$

for some $d-1$-uples $\left(\ell_{1}, \ldots \ell_{j_{0}-1}, \ell_{j_{0}+1}, \ldots, \ell_{d}\right)$ and $\left(\ell_{1}^{\prime}, \ldots \ell_{j_{0}-1}^{\prime}, \ell_{j_{0}+1}^{\prime}, \ldots, \ell_{d}^{\prime}\right)$.

By Lemma 2.9 it follows that

$$
\begin{aligned}
& \left(\ell_{1}-\delta_{1}, \ldots, \ell_{j_{0}-1}-\delta_{j_{0}-1}, \underline{\ell}-\delta_{j_{0}}, \ell_{j_{0}+1}-\delta_{j_{0}+1}, \ldots, \ell_{d}-\delta_{d}\right) \in \mathcal{E}(\Phi), \\
& \left(\ell_{1}^{\prime}-\delta_{1}, \ldots, \ell_{j_{0}-1}^{\prime}-\delta_{j_{0}-1}, \underline{\ell}^{\prime}-\delta_{j_{0}}, \ell_{j_{0}+1}^{\prime}-\delta_{j_{0}+1}, \ldots, \ell_{d}^{\prime}-\delta_{d}\right) \in \mathcal{E}(\Phi) .
\end{aligned}
$$

Thus $\left(\ell_{1}-\ell_{1}^{\prime}, \ldots, \underline{\ell}-\underline{\ell}^{\prime}, \ldots, \ell_{d}-l_{d}^{\prime}\right) \in \mathcal{E}(\Phi)$ with $\underline{\ell}-\underline{\ell}^{\prime} \neq 0$ which completes the proof (for the initial $\Phi$ we have to divide by an integer and obtain a non zero essential value with rational coordinates).

Theorem 3.4. Let $\Phi$ be a rational step cocycle with values in $\mathbb{R}^{d}$. There are $d(\Phi)$, $0 \leq d(\Phi) \leq d$, and a change of basis of $\mathbb{R}^{d}$ given by a rational matrix $M$ such that $M \bar{\Phi}=\left(\hat{\varphi}^{1}, \ldots, \hat{\varphi}^{d(\Phi)}, \hat{\varphi}^{d(\Phi)+1}, \ldots, \hat{\varphi}^{d}\right)$ satisfies:

1) $\mathcal{E}(M \Phi)$ contains the subgroup generated by

$$
(1,0, \ldots, 0),(0,1,0, \ldots, 0), \ldots,(\underbrace{0,0, \ldots, 1}_{d(\Phi)}, 0, \ldots, 0)
$$

2) the cocycle $\hat{\Phi}=\left(\hat{\varphi}^{d(\Phi)+1}, \ldots, \hat{\varphi}^{d}\right)$ is a rational cocycle like (22) and satisfies $\lim _{n}\left\|q_{n} \beta_{j}(\hat{\Phi})\right\|=$ 0 for $j=d(\Phi)+1, \ldots, d$. 
Proof. We will apply successively Lemmas 3.3, 2.11 and 3.2. If $\mathcal{L}\left(\beta_{j}\right)=\{0\}$ for all $j=1, \ldots, d$, we put $d(\Phi)=0$. Suppose not all $\mathcal{L}\left(\beta_{j}\right)$ are equal to $\{0\}$, say $\mathcal{L}\left(\beta_{1}\right) \neq\{0\}$. Then by Lemma 3.3, there is a rational vector $\theta=\left(\theta_{1}, \ldots, \theta_{d}\right) \in \mathcal{E}(\Phi)$ with $\theta_{1} \neq 0$.

Take a linear (rational) isomorphism $M_{1}$ of $\mathbb{R}^{d}$, so that $M_{1}(\theta)=e_{1}$, where $e_{1}=(1,0, \ldots, 0)$ and consider $M_{1}(\Phi)=\left(\varphi_{1}^{\prime}, \ldots, \varphi_{d}^{\prime}\right)$. The step cocycles $\varphi_{2}^{\prime}, \ldots, \varphi_{d}^{\prime}$ have their own representation (22) with $\beta_{j}^{\prime}$ instead of $\beta_{j}$. We now look at $\mathcal{L}\left(\beta_{j}^{\prime}\right)$ for $j=2, \ldots, d$. If all these sets are equal to $\{0\}$, we set $d(\Phi)=1$ and the proof is finished.

Suppose not all $\mathcal{L}\left(\beta_{j}^{\prime}\right)$ for $j=2, \ldots, d$ are equal to zero, say $\mathcal{L}\left(\beta_{2}^{\prime}\right) \neq 0$. We apply Lemma 3.3 to $M_{1}(\Phi)$ and obtain $\theta^{\prime}=\left(\theta_{1}^{\prime}, \theta_{2}^{\prime}, \ldots\right) \in \mathcal{E}\left(M_{1} \Phi\right)$ with $\theta_{2}^{\prime} \neq 0$. Note that $e_{1}$ and $\theta^{\prime}$ are linearly independent. Then consider a linear (rational) isomorphism $M_{2}$ of $\mathbb{R}^{d}$ that fixes $e_{1}$ and sends $\theta^{\prime}$ to $e_{2}$ and set

$$
M_{2}\left(M_{1}(\Phi)\right)=\left(\varphi_{1}^{\prime}, \varphi_{2}^{\prime \prime}, \ldots, \varphi_{d}^{\prime \prime}\right)
$$

(this cocycle has $e_{1}$ and $e_{2}$ as its essential values).

Again, these new cocycles (except for $\varphi_{1}^{\prime}$ ) have their own representation (22) with $\beta_{j}^{\prime \prime}$ for $j=2, \ldots, d$. We now look at $\mathcal{L}\left(\beta_{j}^{\prime \prime}\right)$ for $j=3, \ldots, d$. If the sets $\mathcal{L}\left(\beta_{j}^{\prime \prime}\right), j=3, \ldots, d$, are equal to $\{0\}$ we set $d(\Phi)=2$ and the proof is complete. If not, say $\mathcal{L}\left(\beta_{3}^{\prime \prime}\right) \neq\{0\}$, we obtain a rational vector $\theta^{\prime \prime}=\left(\theta_{1}^{\prime \prime}, \theta_{2}^{\prime \prime}, \theta_{3}^{\prime \prime}, \ldots\right) \in \mathcal{E}\left(M_{2}\left(M_{1}(\Phi)\right)\right)$ with $\theta_{3}^{\prime \prime} \neq 0$. Then consider a (rational) linear isomorphism $M_{3}: \mathbb{R}^{d} \rightarrow \mathbb{R}^{d}$ fixing $e_{1}, e_{2}$ and sending $\theta_{3}^{\prime \prime}$ into $e_{3}$ and pass to the cocycle $M_{3}\left(M_{2}\left(M_{1}(\Phi)\right)\right)$. We complete the proof in finitely many steps.

\subsection{Reduction in the bounded type case}

If we find $d(\Phi)=d$ in Theorem [3.4, then the group $\mathcal{E}(\Phi)$ contains a subgroup with compact quotient in $\mathbb{R}^{d}$ and hence the cocycle $\Phi$ is regular. This is the situation of the following theorem:

Theorem 3.5. Let $\alpha$ be of bounded type. Let $\left(\beta_{1}, \ldots, \beta_{d}\right)$ be such that there is no non trivial rational relation between $1, \alpha, \beta_{1}, \ldots, \beta_{d}$. Then the cocycle $\Phi=\left(1_{\left[0, \beta_{j}\right)}-\beta_{j}\right)_{j=1, \ldots, d}$ is regular. Every step cocycle $\varphi$ with discontinuities at $\left\{0, \beta_{1}, \ldots, \beta_{d}\right\}$ and dimension $d^{\prime} \leq d$ is regular.

Proof. We use the notation of Theorem 3.4. If $d(\Phi)<d$, then we have $\lim _{n}\left\|q_{n} \beta_{j}(\hat{\Phi})\right\|=0$ for $j=d(\Phi)+1, \ldots, d$. As $\alpha$ is of bounded type, taking into account Lemma 3.2 and Lemma 2.4, we find a non trivial rational relation between the numbers $\beta_{j}(\hat{\Phi})$. Since the changes of basis are given by rational matrices $M$, this gives a non trivial rational relation between $1, \alpha, \beta_{1}, \ldots, \beta_{d}$, contrary to the assumption of the theorem. Therefore $d(\Phi)=d$ and the cocycle $\Phi$ is regular. For the second statement, observe that, if $d^{\prime} \leq d$, $\varphi$ is the image of $\Phi$ by a linear map. It is regular if $\Phi$ is regular by Lemma 2.7 .

Remark 8. a) The previous proof is based on the method of rational cocycle, but applies even to non rational cocycle. 
As an illustration of the result, let us consider the cocycle: $\varphi=\theta 1_{[0, \beta)}-1_{[0, \theta \beta)}$, with $(1, \alpha, \beta, \theta \beta)$ rationally independent, $\theta \notin \mathbb{Q}$ and $\beta, \theta \beta \in[0,1)$. This cocycle is not rational, but obtained from the cocycle $\Phi=\left(1_{[0, \beta)}-\beta, 1_{[0, \theta \beta)}-\theta \beta\right)$ by the map $\left(y_{1}, y_{2}\right) \rightarrow \theta y_{1}-y_{2}$. For $\alpha$ of bounded type, $\Phi$ and therefore also $\varphi$ are regular (Theorem [3.5).

b) An example of cocycle for which the previous method does not solve the question of regularity, even for $\alpha$ of bounded type, is $\varphi=1_{[0, \beta)}-1_{[0, \beta)}(.+\gamma)=1_{[0, \beta)}-1_{[0, \beta+\gamma)}+1_{[0, \gamma)}$, obtained from $\Phi=\left(1_{[0, \beta)}-\beta, 1_{[0, \beta+\gamma)}-\beta-\gamma, 1_{[0, \gamma)}-\gamma\right)$ by the map $\left(y_{1}, y_{2}, y_{2}\right) \rightarrow$ $y_{1}-y_{2}+y_{3}$.

(See also Example 2, after Theorem 3.10.)

c) We would like to mention that when $\beta=1 / 2$ and $\alpha$ is of bounded type the regularity (for each $\gamma \in \mathbb{T}$ ) has been shown recently by Zhang [Zh10] using different methods. In fact, Zhang shows that the cocycle $\Phi=\left(1_{[0,1 / 2)}(\cdot)-1 / 2,1_{[0,1 / 2)}(\cdot+\gamma)-1 / 2\right)$ is regular (whenever $\alpha$ is of bounded type).

The regularity of $\Phi$ follows also from Lemma 2.3 and Theorem 3.8 below (see Corollary 3.9).

d) We will give in Subsection 3.4 a different method, based on "clusters" of discontinuity points, which can be applied to the lower dimensional cocycle: $\varphi=1_{[0, \beta)}-1_{[0, \gamma)}-\beta+\gamma$ when $(1, \alpha, \beta, \gamma)$ are rationally dependent.

e) In the bounded type case, the reduction given by Theorem 3.4 reduces to a cocycle of the form (22) such that $\beta_{j} \in \mathbb{Z} \alpha+\mathbb{Z}$ for all $j=d_{1}+1, \ldots, d$. We can even obtain $\beta_{j}=0$ by using the identity: $\alpha=1_{[1-\alpha, 1)}(x)+j(x+\alpha)-j(x)$, for $0<\alpha<1$, where $j(x)=\{x\}$.

\subsection{The case $\left\|q_{n} \beta_{j}\right\| \rightarrow 0$}

If $\left\|q_{n} \beta_{j}\right\| \rightarrow 0, \forall j$ and $\beta_{j} \notin \mathbb{Z} \alpha+\mathbb{Z}$ (a situation which can occur only for $\alpha$ not of bounded type), the previous method of reduction cannot be applied. Nevertheless, there is a first step reduction, based on another method.

Lemma 3.6. Let $\Phi$ be a step function with $D=D(\Phi)$ points of discontinuity. We have $\mu\left(A_{q, \ell}(\Phi)\right)>1-2 D q \varepsilon$, with $\varepsilon=\ell\|q \alpha\|$, where

$$
A_{q, \ell}(\Phi):=\bigcap_{1 \leq s \leq \ell}\left\{x \in \mathbb{T}: \Phi_{q}(x)=\Phi_{q}(x+s q \alpha)\right\}, \ell, q \geq 1 .
$$

Proof. Let $\Delta$ be the set of discontinuities of $\Phi$. If $x \notin A_{q, \ell}(\Phi)$, we can find $s, 1 \leq s<\ell$, and $j, 0 \leq j<q$, such that $\Phi(x+j \alpha) \neq \Phi(x+j \alpha+s q \alpha)$. This implies that $\Phi$ has a discontinuity at $\delta$ on the circle between $x+j \alpha$ and $x+j \alpha+s q \alpha$, and therefore $x$ belongs to the interval $(\delta-j \alpha-\varepsilon, \delta-j \alpha+\varepsilon)$ because $\sup _{1 \leq s \leq \ell}\|s q \alpha\| \leq \ell\|q \alpha\|$. Now, the complement of $A_{q, \ell}(\Phi)$ is included in the set $\bigcup_{0 \leq j<q, t \in \Delta} B(t-j \alpha, \varepsilon)$, whose measure is less than $2 D q \varepsilon$.

Proposition 3.7. Let $\Phi=\left(1_{\left[0, \beta_{j}\right)}-\beta_{j}, j=1, \ldots, d\right)$. Suppose $\beta_{j} \notin \mathbb{Z} \alpha+\mathbb{Z}$ and $\left\|q_{n} \beta_{j}\right\| \rightarrow$ $0, \forall j$, then $\mathcal{E}(\Phi)$ contains a non zero vector in $\mathbb{Z}^{d}$ or a non discrete subgroup of $\mathbb{R}^{d}$. 
Proof. For $n \geq 1$, we can write (cf. 23) $: \varphi_{n}^{j}(x)=\tilde{u}_{(n)}^{j}(x)-\varepsilon\left\|n \beta_{j}\right\|$, where $\varepsilon= \pm 1$ and $\tilde{u}_{(n)}^{j}$ is the integer valued function

$$
\tilde{u}_{(n)}^{j}=u_{(n)}^{j} \text { if }\left\{n \beta_{j}\right\}=\left\|n \beta_{j}\right\|, \quad \tilde{u}_{(n)}^{j}=u_{(n)}^{j}+1 \text { if }\left\{n \beta_{j}\right\}=1-\left\|n \beta_{j}\right\| .
$$

a) If $\mu\left(\left\{x \in \mathbb{T}: \tilde{u}_{\left(q_{n}\right)}^{j_{0}}(x)=0\right\}\right) \not \rightarrow 1$ for some $j_{0}$, the proof is similar to the proof of Lemma 3.3: by passing to a subsequence if necessary to ensure the convergence of all components $\varphi_{q_{n}}^{j}$, we find that $\Phi$ has a quasi-period $\left(\rho_{1}, \rho_{2}, \ldots, \rho_{d}\right)$, with $\rho_{j_{0}} \neq 0$. It follows that $\mathcal{E}(\Phi)$ contains a non-zero vector in $\mathbb{Z}^{d}$.

b) Now, we can assume $\lim _{n} \mu\left(\left\{x \in \mathbb{T}: \tilde{u}_{\left(q_{n}\right)}^{j}(x)=0\right\}\right)=1$, for every $j$.

By Lemma 2.4 there is a sequence $\left(n_{k}\right)$ such that $\left\|q_{n_{k}} \beta_{1}\right\|>\frac{1}{4} q_{n_{k}}\left\|q_{n_{k}} \alpha\right\|$. We put $L_{k}=$ $\left[\eta /\left\|q_{n_{k}} \beta_{1}\right\|\right], k \geq 1$, where $\eta$ is such that $\eta<\frac{1}{16 D}$.

There is at least one index $j_{0}$ such that, infinitely often, $\left\|q_{n_{k}} \beta_{j_{0}}\right\|$ is the biggest value of the set $\left\{\left\|q_{n_{k}} \beta_{j}\right\|, j=1, \ldots, D(\Phi)\right\}$. Hence for $j_{0}$ and an infinite subsequence, still denoted $\left(n_{k}\right)$, we have

$$
0<\left\|q_{n_{k}} \beta_{j}\right\| \leq\left\|q_{n_{k}} \beta_{j_{0}}\right\|, \forall j .
$$

In particular, we have $\left\|q_{n_{k}} \beta_{j_{0}}\right\| \geq \frac{1}{4} q_{n_{k}}\left\|q_{n_{k}} \alpha\right\|$.

Using the notation and the assertion of Lemma 3.6, we have

$$
\mu\left(A_{q_{n_{k}}, L_{k}}(\Phi)\right)>1-2 D q_{n_{k}} L_{k}\left\|q_{n_{k}} \alpha\right\| \geq 1-8 D \eta \geq \frac{1}{2} .
$$

Moreover, using the definition of $A_{q_{n_{k}}, L_{k}}(\Phi)$, for $x \in A_{q_{n_{k}}, L_{k}}(\Phi)$ and $\ell \leq L_{k}$, we have

$$
\Phi_{\ell q_{n_{k}}}(x)=\ell \Phi_{q_{n_{k}}}(x)=\left(\ell \tilde{u}_{\left(q_{\left.n_{k}\right)}\right.}^{j}(x)-\varepsilon \ell\left\|q_{n_{k}} \beta_{j}\right\|, j=1, \ldots, d\right)
$$

with $\varepsilon= \pm 1$. Let $\rho \in(0, \eta)$. Put $\ell_{k}:=\left[\rho /\left\|q_{n_{k}} \beta_{j_{0}}\right\|\right]<\eta /\left\|q_{n_{k}} \beta_{j_{0}}\right\| \leq L_{k}+1$. We have, for $x \in A_{q_{n_{k}}, L_{k}}(\Phi)$, outside of a set of measure tending to 0 ,

$$
\varphi_{\ell_{k} q_{n_{k}}}^{j_{0}}(x)=\ell_{k} \varphi_{q_{n_{k}}}^{j_{0}}(x)=\ell_{k} \tilde{u}_{\left(q_{n_{k}}\right)}^{j_{0}}(x)-\varepsilon \ell_{k}\left\|q_{n_{k}} \beta_{j_{0}}\right\|= \pm\left[\rho /\left\|q_{n_{k}} \beta_{j_{0}}\right\|\right]\left\|q_{n_{k}} \beta_{j_{0}}\right\| \rightarrow \pm \rho .
$$

For the other components $j \neq j_{0}$, outside of a set of measure tending to 0 , we have on $A_{q_{n_{k}}, L_{k}}(\Phi)$

$$
\varphi_{\ell_{k} q_{n_{k}}}^{j}(x)=\ell_{k} \varphi_{q_{n_{k}}}^{j}(x)=\ell_{k} \tilde{u}_{\left(q_{n_{k}}\right)}^{j}(x)-\varepsilon \ell_{k}\left\|q_{n_{k}} \beta_{j}\right\|= \pm\left\|q_{n_{k}} \beta_{j}\right\|\left[\rho /\left\|q_{n_{k}} \beta_{j_{0}}\right\|\right] .
$$

The above quantity is bounded, since $\left\|q_{n_{k}} \beta_{j}\right\|\left[\rho /\left\|q_{n_{k}} \beta_{j_{0}}\right\|\right] \leq \rho\left\|q_{n_{k}} \beta_{j}\right\| /\left\|q_{n_{k}} \beta_{j_{0}}\right\| \leq \rho$. Passing to a subsequence still denoted $\left(n_{k}\right)$ if necessary, we obtain that outside of a set of measure tending to 0 , on $A_{q_{n_{k}}, L_{k}}(\Phi)$ the sequence $\left(\Phi_{\ell_{k} q_{n_{k}}}(x)\right)$ converges to the vector $\left(\rho_{1}, \rho_{2}, \ldots, \rho_{d}\right)$.

Now, the measure of $A_{q_{n_{k}}, L_{k}}(\Phi)$ is bounded away from 0 and the sequence $\left(\ell_{k} q_{n_{k}}\right)$ is a rigidity sequence for $T$, since $\ell_{k} \leq L_{k}+1$ and

$$
L_{k}\left\|q_{n_{k}} \alpha\right\| \leq \eta \frac{\left\|q_{n_{k}} \alpha\right\|}{\left\|q_{n_{k}} \beta_{1}\right\|} \leq 4 \frac{\eta}{q_{n_{k}}} \rightarrow 0
$$


It follows that, for an arbitrary $\rho \in(0, \eta), \mathcal{E}(\Phi)$ contains a vector $\left(\rho_{1}, \rho_{2}, \ldots, \rho_{d}\right) \in \mathcal{E}(\Phi)$, with $\rho_{j_{0}}=\rho$. It follows that $\mathcal{E}(\Phi)$ includes a non-discrete subgroup of $\mathbb{R}^{d}$.

Remark 9. Lemma 3.3 and Proposition 3.7 show that, in dimension 1 , for $\Phi=1_{[0, \beta]}-\beta$, if $\beta \notin \mathbb{Z} \alpha+\mathbb{Z}$, the group $\mathcal{E}(\Phi)$ contains at least a positive integer.

\subsection{Well separated discontinuities, clusters of discontinuities}

The previous method was based on diophantine properties of the values of the integrals for rational cocycle. In this subsection we present results relying on diophantine properties of the discontinuities of the cocycle. We give sufficient conditions for regularity of the cocycle defined by a step function $\Phi: \mathbb{T} \rightarrow \mathbb{R}^{d}$ with integral 0 .

The set of discontinuities of $\Phi_{n}(x)=\sum_{k=0}^{n-1} \Phi(x+k \alpha)$ is $\mathcal{D}_{n}:=\left\{\left\{x_{i}-k \alpha\right\}: 1 \leq i \leq\right.$ $D, 0 \leq k<n\}$. We assume that the points $x_{i}-k \alpha \bmod 1$, for $1 \leq i \leq D, 0 \leq k<n$, are distinct. The jump of $\Phi$ at $x_{i}$ is $\sigma_{i}=\sigma\left(x_{i}\right)=\Phi\left(x_{i}^{+}\right)-\Phi\left(x_{i}^{-}\right)$. A discontinuity of the form $\left\{x_{i}-k \alpha\right\}$ is said to be of type $x_{i}$.

By Lemma 2.1, any interval of the circle of length $\geq 2 / q_{n}$ contains at least one point of the set $\left\{\left\{x_{i}-k \alpha\right\}, k=0, \ldots, q_{n}-1\right\}$, hence at least one discontinuity of $\Phi_{q_{n}}$ of type $x_{i}$ for each $x_{i} \in \mathcal{D}$.

\section{Well separated discontinuities}

We write $\mathcal{D}_{n}=\left\{\gamma_{n, 1}<\ldots<\gamma_{n, D n}<1\right\}$ and $\gamma_{n, D n+1}=\gamma_{n, 1}$, where, for $1 \leq \ell \leq D n$, the points $\gamma_{n, \ell}$ run through the set of discontinuities $\mathcal{D}_{n}$ in the natural order.

Definition 4. The cocycle is said to have well separated discontinuities (wsd), if there is $c>0$ and an infinite set $\mathcal{Q}$ of denominators of $\alpha$ such that

$$
\gamma_{q, \ell+1}-\gamma_{q, \ell} \geq c / q, \forall q \in \mathcal{Q}, \ell \in\{1, \ldots, D q\} .
$$

This condition is similar to Boshernitzan's condition ([Bo85]) for interval exchange transformations. The result below extends an analogous statement when $\Phi$ takes values in $\mathbb{Z}^{d}$ (see CoGu12]).

Theorem 3.8. Let $\Phi$ be a zero mean step function. If $\Phi$ satisfies the wsd property (27), then the group $\mathcal{E}(\Phi)$ includes the set $\left\{\sigma_{i}: i=1, \ldots, D\right\}$ of jumps at discontinuities of $\Phi$. Moreover, $\Phi$ is regular.

Proof. Let us consider $\Phi_{q}(x)$ for $q \in \mathcal{Q}$. By (18) and (25), we can write, with $u_{(q)}^{i, j}(x)$ in a finite fixed set of integers $\mathcal{F}$,

$$
\Phi_{q}=\left(\varphi_{q}^{j}\right)_{j=1, \ldots, d} \text { with } \varphi_{q}^{j}(x)=\sum_{i} t_{i, j} u_{(q)}^{i, j}(x)-\sum_{i} t_{i, j}\left\{q \beta_{i, j}\right\}
$$

Let $\theta^{(q)}:=\left(\theta_{j, q}, j=1, \ldots, d\right)$ with $\theta_{j, q}:=-\sum_{i} t_{i, j}\left\{q \beta_{i, j}\right\}$. We can assume that the limit $\theta:=\lim _{q \rightarrow \infty, q \in \mathcal{Q}} \theta^{(q)}$ exists. The set of values of $\Phi_{q}$ for $q \in \mathcal{Q}$ is included in $R+\theta^{(q)}$ where $R$ is the finite fixed set of vectors $\left\{\left(\sum_{i} t_{i, j} k_{i, j}, j=1, \ldots, d\right): k_{i, j} \in \mathcal{F}\right\}$. 
Let $\mathcal{I}_{q}$ be the partition of the circle into the intervals of continuity of $\Phi_{q}, I_{q, \ell}=\left[\gamma_{q, \ell}, \gamma_{q, \ell+1}\right)$, $1 \leq \ell \leq D q$. With the constant $c$ introduced in (27), let $J_{q, \ell} \subset \mathbb{T}$ be the union of $L:=\lfloor 2 / c\rfloor+1$ consecutive intervals in $\mathcal{I}_{q}$ starting with $I_{q, \ell}$. By (27) every $J_{q, \ell}$ has length $\geq 2 / q$, thus contains an element of the set $\left\{\left\{x_{i}-s \alpha\right\}, s=0, \ldots, q-1\right\}$ for each $x_{i}$.

Therefore, for every jump $\sigma_{i}$ of $\Phi$, there is $v \in R$ and two consecutive intervals $I, I^{\prime} \in \mathcal{I}_{q}$, with $I \cup I^{\prime} \subset J_{q, \ell}$, such that the value of $\Phi_{q}$ is $v+\theta^{(q)}$ on $I$ and $v+\theta^{(q)}+\sigma_{i}$ on $I^{\prime}$.

Given $i \in\{1, \ldots, D\}$, we denote $\mathcal{H}_{q}\left(\sigma_{i}\right)$ the family of intervals $I \in \mathcal{I}_{q}$ such that the jump of $\Phi_{q}$ at the right endpoint of $I$ is $\sigma_{i}$. Since each interval $J_{q, \ell}$ contains an interval $I \in \mathcal{H}_{q}\left(\sigma_{i}\right)$, the cardinality of $\mathcal{H}_{q}\left(\sigma_{i}\right)$ is at least $\frac{q D}{L}$.

Fix additionally $v \in R$ and let $\mathcal{A}_{q}\left(\sigma_{i}, v\right)$ be the set of intervals $I \in \mathcal{H}_{q}\left(\sigma_{i}\right)$ such that $\Phi_{q}(x)=v+\theta^{(q)}$ on $I$. Let $\mathcal{A}_{q}^{\prime}\left(\sigma_{i}, v\right)$ be the set of intervals $I^{\prime} \in \mathcal{I}_{q}$ adjacent on the right to the intervals $I \in \mathcal{A}_{q}\left(\sigma_{i}, v\right)$.

Let $A_{q}\left(\sigma_{i}, v\right)$ be the union of intervals $I \in \mathcal{A}_{q}\left(\sigma_{i}, v\right)$ and $A_{q}^{\prime}\left(\sigma_{i}, v\right)$ the union of intervals $I^{\prime} \in \mathcal{A}_{q}^{\prime}\left(\sigma_{i}, v\right)$. The value of $\Phi_{q}$ is $v+\theta^{(q)}$ on $A_{q}\left(\sigma_{i}, v\right)$ and $v+\theta^{(q)}+\sigma_{i}$ on $A_{q}^{\prime}\left(\sigma_{i}, v\right)$.

There is $v_{0} \in R$ and an infinite subset $\mathcal{Q}_{0}$ of $\mathcal{Q}$ such that, for $q \in \mathcal{Q}_{0}$,

$$
\left|\mathcal{A}_{q}\left(\sigma_{i}, v_{0}\right)\right|,\left|\mathcal{A}_{q}^{\prime}\left(\sigma_{i}, v_{0}\right)\right| \geq \frac{\left|\mathcal{H}_{q}\left(\sigma_{i}\right)\right|}{|R|} \geq \frac{q D}{L|R|} .
$$

By (27) and (28), we have $\mu\left(A_{q}\left(\sigma_{i}, v_{0}\right)\right), \mu\left(A_{q}^{\prime}\left(\sigma_{i}, v_{0}\right)\right) \geq \frac{D c^{2}}{(2+c)|R|}$. Thus $v_{0}+\theta$ and $v_{0}+\theta+\sigma_{i}$ are quasi-periods, hence, by Lemma 2.9 essential values. Since $\mathcal{E}(\Phi)$ is a group, $\sigma_{i}$ is an essential value. Therefore $\mathcal{E}(\Phi)$ includes the group generated by the jumps of $\Phi$.

Finally, notice that the quotient cocycle $\Phi / \mathcal{E}(\Phi)$ is a continuous step cocycle, hence is constant. Therefore, the regularity of $\Phi$ follows from Lemma 2.14.

For $\Phi_{d}:=\left(1_{\left[0, \beta_{j}\right]}-\beta_{j}, j=1, \ldots, d\right)$ with $\beta_{i} \neq \beta_{j}$ whenever $i \neq j$, the jump of $\Phi_{d}$ is $(1, \ldots, 1)$ at 0 and $(0, \ldots, 0,-1,0, \ldots, 0)$ at $\beta_{j}$ ( -1 stands at the $j$-th coordinate), $j=1, \ldots, d$. If the wsd property is satisfied, the group $\mathcal{E}(\Phi)$ includes $\mathbb{Z}^{d}$. Therefore the cocycle $\Phi_{d}$ is regular whenever the wsd property holds.

In view of Lemma 2.3 and Theorem 3.8 , we obtain the following result (where the case $\beta \in \mathbb{Z} \alpha+\mathbb{Z}$ can be treated directly).

Corollary 3.9. Let $\alpha$ be of bounded type. Let $\beta$ be a real number.

1) The cocycle $\left(1_{\left[0, \frac{r}{s}\right)}()-.\frac{r}{s}, 1_{\left[0, \frac{r}{s}\right)}(.+\beta)-\frac{r}{s}\right)$ is regular for every rational number $\frac{r}{s} \in(0,1)$.

2) If $\frac{r_{1}}{s_{1}}, \ldots, \frac{r_{d}}{s_{d}}$ are rational numbers such that $0<\frac{r_{i}}{s_{i}} \beta<1$, then, for every real numbers $t_{1}, \ldots, t_{d}$, the cocycle $\varphi=\sum_{i} t_{i} 1_{\left[0, \frac{r_{i}}{s_{i}} \beta\right)}-\beta \sum_{i} t_{i} \frac{r_{i}}{s_{i}}$ is regular.

\section{Clusters of discontinuities}

For a subset $C$ of discontinuities of $\Phi$, we denote $\sigma(C)=\sum_{x_{i} \in C} \sigma\left(x_{i}\right)$ the corresponding sum of jumps of $\Phi$. The number of discontinuities of $\Phi$ is $D=D(\Phi)$. The following result can be useful when the discontinuities are not well separated. 
Theorem 3.10. Suppose that there are two discontinuities $x_{i_{0}}, x_{j_{0}}$ of $\Phi$ and a subsequence $\left(q_{n_{k}}\right)$ such that for a constant $\kappa>0$ we have

$$
q_{n_{k}}\left\|\left(x_{i_{0}}-x_{j_{0}}\right)-r \alpha\right\| \geq \kappa, \forall|r|<q_{n_{k}} .
$$

Then, if the sum $\sigma(C)$ is $\neq 0$ for each non-empty proper subset $C$ of the set of discontinuities of $\Phi$, then $\Phi$ has a non trivial essential value.

Proof. By Lemma 2.1 any interval of length $2 / q_{n}$ on the circle contains at least one discontinuity of each type $x_{i}$ and at most 4 such discontinuities, therefore at most $4 D(\Phi)$ discontinuities of $\Phi_{q_{n}}$.

Consider the sequence $\mathcal{Q}=\left(q_{n_{k}}\right)$ of denominators satisfying (29). On the circle $\mathbb{T}$ we will deal with families of disjoint intervals of length $4 / q_{n_{k}}$. In fact, we consider families of the form $\left\{I_{j}^{(k)}: j \in J_{k} \subset\left\{0,1, \ldots, q_{n_{k}}-1\right\}\right\}$ with $I_{j}^{(k)}=I_{0}^{(k)}+\{-j \alpha\}$, where $I_{0}^{(k)}=\left[0,4 / q_{n_{k}}\right]$ and $J_{k}$ is such that its cardinality satisfies $\left|J_{k}\right| \geq \delta_{1} q_{n_{k}}$ for a fixed positive constant $\delta_{1}$.

The number of different "patterns of discontinuities" (i.e. consecutive types of discontinuities) which can occur altogether in these intervals is finite (indeed, the length of a pattern of discontinuity is bounded by $8 D(\Phi))$. There are an infinite subsequence of $\mathcal{Q}$ (still denoted by $\mathcal{Q}$ ) and a family of intervals $I_{0}^{(k)}+\{-j \alpha\}, j \in J_{k}^{\prime}$ with $\left|J_{k}^{\prime}\right| \geq \delta_{2} q_{n_{k}}$ for a fixed positive constant $\delta_{2}$ (therefore with a total amount of measure bounded away from 0 ) such that the same pattern of discontinuities occurs in each interval of the family. For illustration, if the cocycle has 4 discontinuities $x_{1}, x_{2}, x_{3}, x_{4}$, we can have for instance in each interval the pattern $\left(x_{1}, x_{3}, x_{4}, x_{3}, x_{2}, x_{1}, x_{2}, x_{4}\right)$, corresponding in a given interval to the "configuration" (a sequence of discontinuities) of the form $\left(\left\{x_{1}-\ell_{j, 1} \alpha\right\},\left\{x_{3}-\right.\right.$ $\left.\left.\ell_{j, 2} \alpha\right\},\left\{x_{4}-\ell_{j, 3} \alpha\right\},\left\{x_{3}-\ell_{j, 4} \alpha\right\},\left\{x_{2}-\ell_{j, 5} \alpha\right\},\left\{x_{1}-\ell_{j, 6} \alpha\right\},\left\{x_{2}-\ell_{j, 7} \alpha\right\},\left\{x_{4}-\ell_{j, 8} \alpha\right\}\right)$.

Now, by taking a further subsequence of $\mathcal{Q}$ if necessary, we will assure a convergence at scale $1 / q_{n_{k}}$ for the discontinuities in $I_{j}^{(k)}$. More precisely, observe that if $\left\{x_{i}-\ell \alpha\right\} \in I_{j}^{(k)}$, then $\left\{x_{i}-\ell \alpha\right\}-\{-j \alpha\} \in I_{0}^{(k)}$. Hence $\left\{x_{i}-(\ell-j) \alpha\right\}$ is in $I_{0}^{(k)}$ and therefore it belongs to the set $\left\{\left\{x_{i}-u \alpha\right\}:|u|<q_{n_{k}}\right\} \cap I_{0}^{(k)}$. Notice that this set $\left\{\left\{x_{i}-u \alpha\right\}:|u|<q_{n_{k}}\right\} \cap I_{0}^{(k)}$ has at least 2 elements and has no more that 8 elements and it does not depend on $j$ (when $k$ changes, the set $J_{k}^{\prime}$ does and so $j$ are different for different $k$, however the common shift, namely the shift by $j \alpha$, leads to points which will be common for all $j \in J_{k}^{\prime}$; on the other hand $r$ runs over a fixed set as the patterns of discontinuities are the same regardless $k$ and $j$ ). Therefore we can write it explicitly as $\left\{\left\{x_{i}-u_{n_{k}, i, r} \alpha\right\}\right\}$.

We can extract a new subsequence of $\mathcal{Q}$ (for which we still keep the same notation $\left.\mathcal{Q}=\left(q_{n_{k}}\right)\right)$ such that for each $\left\{x_{i}-u_{n_{k}, i, r} \alpha\right\}$ the sequence $q_{n_{k}}\left\{x_{i}-u_{n_{k}, i, r} \alpha\right\}$ converges to a limit $y_{i, r} \in[0,4]$ when $k \rightarrow \infty$. This is possible, since there is a finite number of such points in $I_{0}^{(k)}$ for each $n_{k}$.

Therefore the configurations of discontinuities in the intervals $I_{j}^{(k)}$ for $j \in J_{k}^{\prime}$ are converging at the scale $1 / q_{n_{k}}$, i.e. after applying the affinities $x \rightarrow q_{n_{k}}(x-\{-j \alpha\})$. We can group the discontinuities (of type) $x_{i}$ according to the value of the limit $y_{i, r}$.

We call "clusters" the subsets of discontinuity points in $I_{j}^{(k)}$ with the same limit at the scale $q_{n_{k}}$ (hence, such that the corresponding limits $y_{i, r}$ in $[0,4]$ coincide). Observe 
that two discontinuities of the same type $x_{i}$ are at distance $\geq \frac{1}{2 q_{n_{k}}}$ by the point 4 ) of Lemma 2.1 and therefore are not in the same cluster: a cluster contains at most one discontinuity of a given type $x_{i}$. In view of (29), the number of elements in a cluster is strictly less than $D(\Phi)$ the number of discontinuities of $\Phi$.

By passing once more to a subsequence of $\mathcal{Q}$ (still denoted by $\mathcal{Q}=\left(q_{n_{k}}\right)$ ) if necessary, we extract a sequence of families of disjoint "good" intervals of length $4 / q_{n_{k}}$ with the same configuration of clusters inside the intervals of a family. There are at least three different clusters in each "good" interval (since for an interval of length $4 / q_{n_{k}}$ a given type of discontinuity occurs at least twice and must occur in different clusters as shown above, moreover the number of elements in a cluster is at most $D(\Phi)-1)$. The clusters in each interval are separated by more than $c / q_{n_{k}}$. As in the proof of Theorem 3.8, the values of the cocycle at time $q_{n_{k}}$ are $v+\theta^{\left(q_{n_{k}}\right)}$ with $v$ in a fixed finite set and $\left(\theta^{\left(q_{n_{k}}\right)}\right)$ a converging sequence.

For $k$ large, clusters of discontinuities are separated by intervals of order $c_{1} / q_{n_{k}}$ for a fixed positive constant $c_{1}$ and there are at least 3 clusters in a "good" interval $I_{j}^{(k)}$. The number of intervals in the families is greater than a fixed fraction of $q_{n_{k}}$. It follows that, under the assumption that the sum of jumps $\sigma(C)$ is $\neq 0$ for each non-empty proper subset $C$ of the set of discontinuities of $\Phi$, the cocycle at time $q_{n_{k}}$ is close to a non zero constant on a set which has a measure bounded away from 0 .

Therefore there $\Phi$ has a non trivial quasi-period, hence a non trivial finite essential value.

Recall that, by Remark 7, if $x_{1}, \ldots, x_{D}$ are all discontinuities of a step cocycle $\Phi$, then for $i \neq j$ we can assume that $x_{i}-x_{j}$ is not a multiple of $\alpha$ modulo 1 . Assume that $\alpha$ is of bounded type. Then, fixing $i_{0} \neq j_{0}$ and using Lemma 2.3 to select a subsequence $\left(q_{n_{k}}\right)$ so that (29) holds for a constant $\kappa>0$, the assumption of the theorem are fulfilled.

\section{Example 1: cocycle with 3 discontinuities}

Let $\alpha$ be an irrational number of bounded type. Let $\varphi$ be a scalar cocycle with 3 effective discontinuities $0, \beta, \gamma$. The sum of jumps for the 3 discontinuities is 0 , and for subsets of 1 or of 2 discontinuities it is always non zero. If $\beta$ (resp. $\gamma$ ) is not in $\mathbb{Z} \alpha+\mathbb{Z}$, by Lemma 2.3 there are subsequences of denominators along which the discontinuities of type $\beta$ (resp. $\gamma$ ) belong to clusters which reduce to a single discontinuity or to two discontinuities. Therefore, by Theorem 3.10 the group of finite essential values does not reduce to $\{0\}$.

\section{Example 2: cocycle with 4 discontinuities}

Let us consider the $\mathbb{R}$-valued cocycle $a\left(1_{[0, \beta)}(\cdot)-\beta\right)-\left(1_{[0, \beta)}(\cdot-\gamma)-\beta\right)$ with $\beta<\gamma$.

There are 4 discontinuity points: $(0, \beta, \gamma, \beta+\gamma)$ with respective jumps $+a,-a,-1,+1$. Assume that $\beta$ is such that there is a subsequence $\left(q_{n_{k}}\right)$ and a constant $\kappa>0$ such that

$$
q_{n_{k}}\|\beta-r \alpha\| \geq \kappa, \forall|r|<q_{n_{k}} .
$$


We apply the method of Theorem 3.10 , with the subsequence $\left(q_{n_{k}}\right)$. By the above condition on $\beta$, in a cluster we can find either a single discontinuity, or two discontinuities of type in $(0, \gamma),(0, \beta+\gamma),(\beta, \gamma),(\beta, \beta+\gamma)$ with respective sum of jumps: $a-1, a+1$, $-(a+1),-a+1$. The case of 3 discontinuities is excluded.

Therefore, if $a \notin\{ \pm 1\}$, we have a non trivial essential value. When $a=-1$, then the cocycle reads $-1_{[0, \beta)}(\cdot)-1_{[0, \beta)}(\cdot-\gamma)+2 \beta$, and by Theorem 3.5 or the method of Proposition 3.7 we obtain a non trivial essential value.

So for the classification of the cocycle $a\left(1_{[0, \beta)}(\cdot)-\beta\right)-\left(1_{[0, \beta)}(\cdot-\gamma)-\beta\right)$ the only case to be considered is $a=1$. This leaves open the question of the regularity of the cocycle $1_{[0, \beta)}(\cdot)-\left(1_{[0, \beta)}(\cdot-\gamma)\right.$ for $\alpha$ of bounded type and any $\beta, \gamma$.

\subsection{On the regularity of $\Phi_{d}, d=1,2,3$}

$\mathbf{d}=\mathbf{1}, \Phi_{1}=1_{[0, \beta)}-\beta$

Theorem 3.11. The cocycle $\Phi_{\beta}=1_{[0, \beta)}-\beta$ is regular over any irrational rotation.

Proof. If $\beta \in \mathbb{Z} \alpha+\mathbb{Z}$, then $\Phi_{\beta}$ is a coboundary (see Remark 7 ). Suppose that $\beta \notin \mathbb{Z} \alpha+\mathbb{Z}$. Then, by Lemma 3.3 and Proposition 3.7 , there is a positive integer in the group $\mathcal{E}(\Phi)$ (cf. Remark 9). Therefore the cocycle $\Phi_{\beta}$ is always regular.

Remark 10. If $\beta, \alpha, 1$ are independent over $\mathbb{Q}$, then by a result of Oren (Or83) the cocycle defined by $\Phi_{\beta}$ is ergodic.

$\mathbf{d}=\mathbf{2}, \Phi_{2}=\left(1_{[0, \beta)}-\beta, 1_{[0, \gamma)}-\gamma\right)$

\section{a) $\alpha$ of bounded type}

Theorem 3.12. If $\alpha$ is of bounded type, the cocycle $\Phi_{2}=\left(1_{[0, \beta)}-\beta, 1_{[0, \gamma)}-\gamma\right)$ is regular.

Proof. Recall that we constantly assume that $\beta, \gamma, \beta-\gamma$ are not in $\mathbb{Z} \alpha+\mathbb{Z}$. The proof is done in three steps:

Step 1. $\mathcal{E}\left(\Phi_{2}\right) \neq\{0\}$; indeed, this follows immediately from the proof of Theorem 3.4 applied to $\beta \notin \mathbb{Z} \alpha+\mathbb{Z}$ (see Lemma 2.4 and Lemma 3.3).

Step 2. $\beta=\gamma$; then our cocycle is regular by Theorem 3.11 .

Step 3. $0<\beta<\gamma<1$. Now, we claim that for each $a, b \in \mathbb{R}$ the cocycle $a\left(1_{[0, \beta)}-\beta\right)+$ $b\left(1_{[0, \gamma)}-\gamma\right)$ is regular. Indeed, we have already noticed this property to hold if $a$ or $b$ is equal to zero. When $a \neq 0 \neq b$, we obtain a step cocycle with 3 effective discontinuities $0, \beta$ and $\gamma$. In that case we apply Theorem 3.10 (see the application to cocycles with 3 discontinuities, example 1 after the proof) to conclude that our scalar cocycle has a non-zero finite essential value and hence is regular. The claim immediately follows. The regularity of $\Phi$ is now an immediate consequence of Corollary 2.13. 
Remark 11. Notice that we can apply other previous results to obtain another, more complex proof of Theorem 3.12, which however can be applied in other situations. Indeed, since $\alpha$ of bounded type, we apply Theorem 3.5 to conclude that the cocycle $\Phi$ is regular whenever $\beta, \gamma, \alpha, 1$ are independent over $\mathbb{Q}$.

Otherwise, there are integers $r, s, v, w$ not all equal to zero such that $r \beta+s \gamma+v \alpha+w=0$.

The case when $\beta$ or $\gamma$ belongs to $\mathbb{Z} \alpha+\mathbb{Z}$ is excluded (cf. Remark [7).

1) Assume that $\beta, \gamma \notin \mathbb{Q} \alpha+\mathbb{Q}$ and $\beta-\gamma \notin \mathbb{Q} \alpha+\mathbb{Q}$.

If $s$ or $r \neq 0$, say $s \neq 0$ then $\gamma=-\frac{r}{s} \beta-\frac{v}{s} \alpha-\frac{w}{s}$. We apply Lemma 2.3 for $\beta_{1}=\frac{1}{1} \beta+\frac{0}{1} \alpha+\frac{0}{1}$, $\beta_{2}=\frac{-r}{s} \beta+\frac{-v}{s} \alpha+\frac{-w}{s}$ and $\beta_{3}=\frac{-r-s}{s} \beta+\frac{-v}{s} \alpha+\frac{-w}{s}$ and obtain a subsequence $\left(q_{n_{k}}\right)$ along which the wsd property is satisfied for the discontinuities of $\Phi_{2}$. Then Theorem 3.8 applies.

2) Suppose $s=0$ and $\gamma \notin \mathbb{Q} \alpha+\mathbb{Q}, \beta \in \mathbb{Q} \alpha+\mathbb{Q}$. It is enough to show that $d_{1}=2$ in Theorem 3.4. By the proof of that theorem applied to $\beta \notin \mathbb{Z} \alpha+\mathbb{Z}$, in view of Lemma 2.4, we obtain $M: \mathbb{R}^{2} \rightarrow \mathbb{R}^{2}$ a rational change of coordinates such that $M \Phi_{2}=\left(\psi^{1}, \psi^{2}\right)$ has $(1,0)$ as its essential value. On the other hand, by Lemma 3.2 (taking into account that $\operatorname{det} M \neq 0$ ) and remembering that under our assumption $\beta$ and $\gamma$ are independent over $\mathbb{Q}$, we obtain that $\beta\left(\psi^{i}\right) \notin \mathbb{Z} \alpha+\mathbb{Z}, i=1,2$. Therefore, again by Lemma 2.4, $\mathcal{L}\left(\beta\left(\psi^{i}\right)\right) \neq\{0\}$, hence by the proof of Theorem 3.4, $d_{1}=2$.

3) The missing case $\beta-\gamma \in \mathbb{Q} \alpha+\mathbb{Q}$ (see the assumption in 1) and the separate case $\beta, \gamma \in \mathbb{Q} \alpha+\mathbb{Q})$ are covered by Lemma 2.3 and an application of Theorem 3.8 .

\section{b) $\alpha$ of non bounded type}

For $d=2$ and $\alpha$ not of bounded type the question of construction of a non regular step function is not solved and the purpose of this paragraph is to present some observations.

From Lemma 3.3 and Proposition 3.7, we know that $\mathcal{E}(\Phi)$ does not reduce to $\{0\}$. By Corollary 2.13, the regularity of the cocycle is equivalent to the regularity of the one dimensional cocycles with 3 discontinuities: $\varphi=a\left(1_{[0, \beta)}-\beta\right)-b\left(1_{[0, \gamma)}-\gamma\right)$, where $a, b$ are arbitrary real numbers. Since we know already that regularity holds for $b=0$, it suffices to consider $\varphi=a 1_{[0, \beta)}-1_{[0, \gamma)}-(a \beta-\gamma)$.

It is interesting to understand the particular case $\gamma=\ell \beta$, with $\ell$ a positive integer. We will give some partial results on this cocycle and ask questions.

First of all, there are special situations where one can conclude that the cocycle $\varphi=$ $\ell 1_{[0, \beta)}-1_{[0, \ell \beta)}$ is a coboundary (we assume that $\ell \beta<1$ ). We use the following result of Guenais and Parreau (with the notation of Section 2.1, in particular $T x=x+\alpha$ ):

Theorem 3.13. (GuPa06], Theorem 2) Let $\varphi$ be a step function on $\mathbb{T}$ with integral 0 and jumps $-s_{j}$ at distinct points $\left(\beta_{j}, 0 \leq j \leq m\right), m \geq 1$, and let $t \in \mathbb{T}$. Suppose that there is a partition $\mathcal{P}$ of $\{0, \ldots, m\}$ such that for every $J \in \mathcal{P}$ and $\beta_{J} \in\left\{\beta_{j}: j \in J\right\}$ :

(i) $\sum_{j \in J} s_{j} \in \mathbb{Z}$; 
(ii) for every $j \in J$, there is a sequence of integers $\left(b_{n}^{j}\right)_{n}$ such that

$$
\beta_{j}=\beta_{J}+\sum_{n \geq 0} b_{n}^{j} q_{n} \alpha \quad \bmod 1 \text {, with } \sum_{n \geq 0} \frac{\left|b_{n}^{j}\right|}{a_{n+1}}<+\infty \text { and } \sum_{n \geq 0}\left\|\sum_{j \in J} b_{n}^{j} s_{j}\right\|^{2}<+\infty ;
$$

(iii) there is an integer $k^{\prime}$ such that $t=k^{\prime} \alpha-\sum_{J \in \mathcal{P}} t_{J}$ where

$$
t_{J}=\beta_{J} \sum_{j \in J} s_{j}+\sum_{n \geq 0}\left[\sum_{j \in J} b_{n}^{j} s_{j}\right] q_{n} \alpha \bmod 1 .
$$

Then there is a measurable function $f$ of modulus 1 solution of

$$
e^{2 i \pi \varphi}=e^{2 i \pi t} f \circ T / f \text {. }
$$

Conversely, when $\sum_{j \in J} s_{j} \notin \mathbb{Z}$ for every proper non empty subset $J$ of $\{0, . ., m\}$, these conditions are necessary for the existence of a solution of (31).

Take $\varphi=\ell 1_{[0, \beta]}-1_{[0, \ell \beta]}$. With the previous notation, the discontinuities are at $\beta_{0}=$ $0, \beta_{1}=\beta, \beta_{2}=\gamma=\ell \beta(m=2)$ with jumps $\ell-1,-\ell, 1$ respectively and the partition $\mathcal{P}$ is the trivial partition with the single atom $J=\{0,1,2\}$. We also have $\beta_{J}=0$, $\sum_{j \in J} s_{j}=0$.

Suppose that the parameter $\beta$ has an expansion in base $\left(q_{n} \alpha\right)$ (Ostrowski expansion, see [IN88]):

$$
\beta=\sum_{n \geq 0} b_{n} q_{n} \alpha \bmod 1, \text { with } \sum_{n \geq 0} \frac{\left|b_{n}\right|}{a_{n+1}}<+\infty, b_{n} \in \mathbb{Z} .
$$

We can take $b_{n}^{0}=0, b_{n}^{1}=b_{n}, b_{n}^{2}=\ell b_{n}$, so that $\sum_{j \in J} b_{n}^{j} s_{j}=\ell b_{n}-\ell b_{n}=0$. In view of Theorem 3.13, for every real $s$, the multiplicative equation $e^{2 \pi i s \varphi}=f \circ T / f$ has a measurable solution $f: \mathbb{T} \rightarrow \mathbb{S}^{1}$. By using Theorem 6.2 in [MoSc80], we conclude that $\varphi$ is a measurable coboundary. Let us mention that another proof based on the tightness of the cocycle $\left(\varphi_{n}\right)$ can also be given.

Conversely, if $\varphi$ is a measurable coboundary, then $e^{2 \pi i s \varphi}=f \circ T / f$, for $s$ real has a measurable solution, and this implies that $\beta$ has the expansion given by (32).

Therefore we obtain:

Proposition 3.14. If $\ell$ is a positive integer with $\ell \beta<1$, then the cocycle $\varphi=\ell 1_{[0, \beta)}$ $1_{[0, \ell \beta)}$ is a coboundary if and only if $\beta$ satisfies (32).

Question: A question is to know if the cocycle $\varphi=\ell 1_{[0, \beta]}-1_{[0, \ell \beta)}$ is regular or not, when $\beta$ has an expansion $\beta=\sum_{n \geq 0} b_{n} q_{n} \alpha \bmod 1$, with $\lim _{n} \frac{\left|b_{n}\right|}{a_{n+1}}=0$ and $\sum_{n \geq 0} \frac{\left|b_{n}\right|}{a_{n+1}}=+\infty$. (Notice that by Theorem 3.13 it cannot be a coboundary.)

$\mathbf{d}=\mathbf{3}, \Phi_{3}=\left(1_{[0, \beta)}-\beta, 1_{[0, \gamma)}-\gamma, 1_{[0, \delta)}-\delta\right)$

We will consider $\alpha$ of non bounded type and construct non regular cocycles (cf. [Co09]). For $r \in \mathbb{R}$, we denote by $\rho_{r}$ the translation $x \rightarrow x+r \bmod 1$. 
Theorem 3.15. Assume that $T x=x+\alpha$ on the circle $\mathbb{T}$. If $\alpha$ is not of bounded type, then there exists $\beta$ such that $\varphi=1_{[0, \beta)}-1_{[0, \beta)} \circ \rho_{r}$ is a non regular cocycle for $r$ in a set of full Lebesgue measure.

Proof. By a result of Merril ([Me85], Theorem 2.5 therein, see also Theorem 3.13 above from [GuPa06]), we know that, if $\beta$ satisfies (32), then there is an uncountable set of real numbers $s$ (so containing irrational numbers) such that we can solve the following quasi-coboundary multiplicative equation in $(s, \beta)$ : for $s \in \mathbb{R}$ there exist $|c|=1$ and a measurable function $f: \mathbb{T} \rightarrow \mathbb{S}^{1}$ such that $e^{2 \pi i s 1_{[0, \beta)}}=c f / f \circ T$.

For this choice of $\beta$ and $s$ ( $s$ is irrational), $e^{2 \pi i s\left(1_{[0, \beta)}-1_{[0, \beta)} \rho^{\circ}\right)}$ is a multiplicative coboundary for every $r$.

For the integer valued cocycle $\psi_{r}=1_{[0, \beta)}-1_{[0, \beta)} \circ \rho_{r}$ we obviously have $\mathcal{E}\left(\psi_{r}\right) \subset \mathbb{Z}$. On the other hand, $s \psi_{r}(x)=n(x)+F(x)-F(x+\alpha)$, with $F: X \rightarrow \mathbb{R}$ and $n(\cdot): X \rightarrow \mathbb{Z}$ measurable. Therefore $\psi_{r}(x)=s^{-1} n(x)+s^{-1} F(x)-s^{-1} F(x+\alpha)$.

It follows that the group of finite essential values over $T$ of the cocycle $\psi_{r}$ is also included in the group $\frac{1}{s} \mathbb{Z}$ and therefore $\overline{\mathcal{E}}\left(\psi_{r}\right) \subset\{0, \infty\}$.

This implies that $\psi_{r}$ is either non regular or a coboundary (cf. Subsection 2.2). The latter case cannot occur for a set of values of $r$ of positive measure, because otherwise, by Proposition 3.16 below, $1_{[0, \beta)}-\beta$ is an additive coboundary up to some additive constant $c$ (and necessarily $c=0$, since the cocycle defined by $1_{[0, \beta)}-\beta$ is recurrent). But this would imply that $e^{2 \pi i \beta}$ is an eigenvalue of the rotation by $\alpha$, a contradiction.

Therefore the cocycle $1_{[0, \beta)}-1_{[0, \beta)} \circ \rho_{r}$ is non regular for a.e. $r \in \mathbb{R}$.

Proposition 3.16. Assume that $K$ is a compact connected Abelian (monothetic) group . Let $T$ be an ergodic rotation on $K$. Let $\varphi: K \rightarrow \mathbb{R}$ be a cocycle. Assume moreover, than on a set of $g \in K$ of positive Haar measure we can find a measurable function $\psi_{g}: K \rightarrow \mathbb{R}$ such that

$$
\varphi-\varphi(g+\cdot)=\psi_{g} \circ T-\psi_{g}
$$

Then $\varphi$ is an additive quasi-coboundary, i.e. $\varphi=b+h \circ T-h$, for a measurable function $h: K \rightarrow \mathbb{R}$ and a constant $b \in \mathbb{R}$.

Proof. For $g \in K$ satisfying (33) and arbitrary $s \in \mathbb{R}$ we have:

$$
\frac{e^{2 \pi i s \varphi(x)}}{e^{2 \pi i s \varphi(g+x)}}=\frac{e^{2 \pi i s \psi_{g}(T x)}}{e^{2 \pi i s \psi_{g}(x)}}
$$

According to Proposition 3 in [Le93, for every $s$ there exist $\lambda_{s}$ with $\left|\lambda_{s}\right|=1$ and a measurable function $\zeta_{s}: X \rightarrow \mathbb{S}^{1}$ such that $e^{2 \pi i s \varphi}=\lambda_{s} \cdot \zeta_{s} \circ T / \zeta_{s}$. By Theorem 6.2 in [MoSc80, the result follows. 
Remark 12. 1) If $\beta$ satisfies (32), then either $1_{[0, \beta)}-1_{[0, \beta)} \circ \rho_{r}$ is non regular or is a coboundary. We have shown that the latter case can occur only for $r$ in a set of zero measure. A problem is to explicit values of $r$ for which $1_{[0, \beta)}-1_{[0, \beta)} \circ \rho_{r}$ is not a coboundary.

2) If $\psi_{\beta, \frac{1}{2}}:=1_{[0, \beta)}-1_{[0, \beta)} \circ \rho_{\frac{1}{2}}$ is non regular, then $\psi_{\left[\frac{1}{2}-\beta, \frac{1}{2}\right)}:=1_{\left[0, \frac{1}{2}-\beta\right)}-1_{\left[0, \frac{1}{2}-\beta\right)} \circ \rho_{\frac{1}{2}}$ is regular. Indeed the sum of these two cocycles is $1_{\left[0, \frac{1}{2}\right)}-1_{\left[\frac{1}{2}, 1\right)}$. It can be easily shown that this latter cocycle has non trivial quasi periods. The non regularity of $\psi_{\beta, \frac{1}{2}}$ implies that $\left(\psi_{\left[\beta, \frac{1}{2}\right)}\right)_{q_{n}}$, the cocycle at times $q_{n}$, tends to 0 in probability, so that $\psi_{\left[\frac{1}{2}-\beta, \frac{1}{2}\right)}$ has non trivial quasi periods.

Corollary 3.17. There are values of the parameters $(\beta, \gamma, \delta)$ such that

$$
\Phi_{3}=\left(1_{[0, \beta)}-\beta, 1_{[0, \gamma)}-\gamma, 1_{[0, \delta)}-\delta\right)
$$

is non regular.

Proof. Suppose that $0<\beta<\gamma<\delta$ and $\delta=\beta+\gamma$. By applying the map $\left(y_{1}, y_{2}, y_{3}\right) \rightarrow$ $y_{1}+y_{2}-y_{3}$, we obtain the 1 -dimensional cocycle $1_{[0, \beta)}(\cdot)-1_{[0, \beta)}(\cdot+\gamma)$, which is non regular by Theorem 3.15 for a value of the parameter $\beta$ satisfying (32) and almost all $\gamma$. Lemma 2.7 implies the non regularity of $\Phi_{3}$ for these values of the parameters.

Note that for $d=2$, i.e. for two parameters $(\beta, \gamma)$, an attempt to obtain a non regular cocycle is to take $\gamma=2 \beta$ and the linear combination: $2\left(1_{[0, \beta)}(\cdot)-\beta\right)-\left(1_{[0,2 \beta)}(\cdot)-2 \beta\right)=$ $1_{[0, \beta)}(\cdot)-1_{[0, \beta)}(\cdot+\beta)$. We obtain the cocycle discussed above (cf. Proposition 3.14) and the question previously mentioned above is whether there are values of $\beta$ such that it is non regular.

\section{Application to affine cocycles}

We consider now the affine cocycle

$$
\Psi_{d+1}(x):=\left(\psi(x), \psi\left(x+\beta_{1}\right), \ldots, \psi\left(x+\beta_{d}\right)\right), \text { where } \psi(x)=\{x\}-\frac{1}{2} .
$$

\subsection{Reduction to a step function}

By a straightforward calculation we have the following formula for the cocycle $\psi$ :

$$
\psi_{q_{n}}(x)=q_{n} x+\frac{q_{n}\left(q_{n}-1\right)}{2} \alpha-\frac{q_{n}}{2}+M(x),
$$

where $M$ is a (non 1 -periodic) function with values in $\mathbb{Z}$. It follows that, for $\beta \in[0,1)$,

$$
\psi_{q_{n}}(\{x+\beta\})
$$




$$
= \begin{cases}\psi_{q_{n}}(x)+q_{n} \beta+(M(x+\beta)-M(x)), & \text { if } \quad x+\beta<1, \\ \psi_{q_{n}}(x)+\left(q_{n} \beta-q_{n}\right)+(M(\{x+\beta\})-M(x)), & \text { if } \quad 1 \leqslant x+\beta<2 .\end{cases}
$$

We will reduce the cocycle $\Psi_{d+1}$ to step cocycles using the group of finite essential values.

Proposition 4.1. The group $\mathcal{E}\left(\Psi_{d+1}\right)$ includes $\Delta_{d+1}=\{(t, \ldots, t): t \in \mathbb{R}\}$, the diagonal subgroup of $\mathbb{R}^{d+1}$.

Proof. Denote $S_{i}(x)=\rho_{\beta_{i}}(x)=x+\beta_{i} \bmod 1$. Suppose that $\left\{q_{n_{k}} \beta_{i}\right\} \rightarrow c_{i}$, with $c_{i} \in[0,1)$ for $i=1, \ldots, d$, and consider the measures

$$
\nu_{k}:=\left(\left(\psi \times \psi \circ S_{1} \times \ldots \times \psi \circ S_{d}\right)_{q_{n_{k}}}\right)_{*}(\mu), \quad k \geq 1
$$

Since

$$
\forall x, y \in[0,1), \quad\left|\psi_{q_{n_{k}}}(x)-\psi_{q_{n_{k}}}(y)\right|<2 V(\psi)=2
$$

and $\int \psi d \mu=0$, we have that $\operatorname{Im}\left(\psi \times \psi \circ S_{1} \times \ldots \times \psi \circ S_{d}\right)_{q_{n_{k}}} \subset[-2,2]^{d+1}$, so that $\nu_{k}$ is concentrated on $[-2,2]^{d+1}$.

It follows that we can select a subsequence of $\left(\nu_{k}\right)$ (still denoted $\left(\nu_{k}\right)$ ) which converges to a probability measure $\nu$ concentrated on $[-2,2]^{d+1}$. We will show in what kind of a subset of $\mathbb{R}^{d+1}$ the support of $\nu$ is included. Consider the image of the measure $\nu_{k}$ via

$$
F: \mathbb{R}^{d+1} \rightarrow \mathbb{R}^{d}, \quad F\left(x_{0}, \ldots, x_{d}\right)=\left(x_{1}-x_{0}, \ldots, x_{d}-x_{0}\right) .
$$

In view of (35), we obtain

$$
F \circ\left(\psi \times \psi \circ S_{1} \times \ldots \times \psi \circ S_{d}\right)_{q_{n_{k}}}(x)=\left(\left\{q_{n_{k}} \beta_{1}\right\}+M_{1}(x), \ldots,\left\{q_{n_{k}} \beta_{d}\right\}+M_{d}(x)\right)
$$

with $M_{i}(x) \in \mathbb{Z}$, whence $F_{*} \nu_{k}$ is the measure concentrated on $\left(\left\{q_{n_{k}} \beta_{1}\right\}, \ldots,\left\{q_{n_{k}} \beta_{d}\right\}\right)+\mathbb{Z}^{d}$. Since $\nu_{k} \rightarrow \nu$ weakly, $F_{*} \nu_{k} \rightarrow F_{*} \nu$ (because all these measures are concentrated on a bounded subset of $\left.\mathbb{R}^{d+1}\right)$. As $\left\{q_{n_{k}} \beta_{i}\right\} \rightarrow c_{i}$, it follows that

$$
\operatorname{supp} \nu \subset\left\{\left(x_{0}, \ldots, x_{d}\right) \in \mathbb{R}^{d+1}: x_{i}-x_{0}=c_{i}+k_{i}, k_{i} \in \mathbb{Z}, i=1, \ldots, d\right\} .
$$

The set on the right hand side of this inclusion is equal to the union of sets of the form $\left\{\left(x, x-\left(c_{1}+k_{1}\right), \ldots, x-\left(c_{d}+k_{d}\right): x \in \mathbb{R}\right\}\right.$, hence of countably many lines parallel to the diagonal $\Delta_{d+1}$. Moreover, the support of $\nu$ is uncountable (because one dimensional projections of $\nu$ are absolutely continuous measures - see [LePaVo96]), whence it must be uncountable on one of these lines. In view of Proposition 2.8 , $\operatorname{supp} \nu \subset \mathcal{E}\left(\Psi_{d+1}\right)$ and since $\mathcal{E}\left(\Psi_{d+1}\right)$ is a group, we have $\operatorname{supp} \nu-\operatorname{supp} \nu \subset \mathcal{E}\left(\Psi_{d+1}\right)$. However, the set $\Delta_{d+1} \cap(\operatorname{supp} \nu-\operatorname{supp} \nu)$ is uncountable, so because $\mathcal{E}\left(\Psi_{d+1}\right)$ is closed, we must have $\Delta_{d+1} \subset \mathcal{E}\left(\Psi_{d+1}\right)$ and the proof is complete.

Corollary 4.2. $\left(\psi, \psi \circ S_{1}, \ldots, \psi \circ S_{d}\right)$ is ergodic whenever the set of accumulation points of $\left(\left\{q_{n} \beta_{1}\right\}, \ldots,\left\{q_{n} \beta_{d}\right\}\right)$ is dense in $\mathbb{T}^{d}$. 
Proof. From the proof of Proposition 4.1 it follows that with every accumulation point $\left(c_{1}, \ldots, c_{d}\right)$ of $\left(\left\{q_{n} \beta_{1}\right\}, \ldots,\left\{q_{n} \beta_{d}\right\}\right)$ we obtain a line $\left\{\left(x, x-\left(c_{1}+k_{1}\right), \ldots, x-\left(c_{d}+k_{d}\right)\right.\right.$ : $x \in \mathbb{R}\}$ (and the smallest subgroup in which the line is included) which is included in the group of essential values. Since the set of accumulation points is dense and $\mathcal{E}\left(\Psi_{d+1}\right)$ is closed, it follows that the only possibility is that $\mathcal{E}\left(\Psi_{d+1}\right)=\mathbb{R}^{d+1}$ which is equivalent to the fact that $\Psi_{d+1}$ is ergodic.

By Lemma 2.6 the study of $\Psi_{d+1}$ reduces to that of the quotient cocycle $\Psi_{d+1}+\Delta_{d+1}$ : $\mathbb{T} \rightarrow \mathbb{R}^{d+1} / \Delta_{d+1}$. Using the epimorphism $\mathbb{R}^{d+1} \ni\left(y_{0}, \ldots, y_{d}\right) \rightarrow\left(y_{1}-y_{0}, \ldots, y_{d}-y_{0}\right) \in \mathbb{R}^{d}$ (whose kernel is equal to $\Delta_{d+1}$ ), the quotient is given by the cocycle

$$
\Phi_{d}(x)=\left(1_{\left[0,1-\beta_{j}\right)}-1+\beta_{j}\right)_{j=1, \ldots, d} .
$$

\subsection{Small values of $d=1,2,3\left(\right.$ and $\left.\Psi_{d+1}\right)$}

1) $d=1, \Psi_{2}=(\psi(x), \psi(x+\beta))$

Applying Theorem 4.1 and Lemma 2.6 we can reduce the cocycle $\Psi_{2}$ to the quotient cocycle $\left(\Psi_{2}+\Delta_{2}\right)(x)=1_{[0,1-\beta)}-1+\beta$. We conclude using Theorem 3.11 that $\Psi_{2}$ is regular over any irrational rotation $T$.

2) $d=2, \Psi_{3}=(\psi(x), \psi(x+\beta), \psi(x+\gamma))$

As above we reduce the cocycle $\Psi_{3}$ to the quotient cocycle $\left(\Psi_{3}+\Delta_{3}\right)(x)=\left(1_{[0,1-\beta)}-1+\right.$ $\left.\beta, 1_{[0,1-\gamma)}-1+\gamma\right)$. Recall that we have seen in subsection 3.5 that for $\alpha$ with bounded partial quotients $\Psi_{3}+\Delta_{3}$ is regular and therefore the affine cocycle is also regular when $\alpha$ has bounded partial quotients.

3) $d=3, \Psi_{4}=(\psi(x), \psi(x+\beta), \psi(x+\gamma), \psi(x+\delta))$

Theorem 4.3. There are values of the parameters $(\beta, \gamma, \delta)$ for which the cocycle is non regular.

Proof. After reduction by $\Delta_{4}$, the result follows from Corollary 3.17 .

\subsection{Ergodicity is generic}

We consider, as before, the cocycle $\psi(x)=\{x\}-\frac{1}{2}$ and let $S_{\beta}(x)=x+\beta$ be the rotation by $\beta \in[0,1)$ on $\mathbb{T}$.

Proposition 4.4. The set $\left\{\left(\beta_{1}, \ldots, \beta_{d}\right) \in \mathbb{T}^{d}:\left(\psi, \psi \circ S_{\beta_{1}}, \ldots, \psi \circ S_{\beta_{d}}\right)\right)$ is ergodic $\}$ is residual. 
Proof. Using Corollary 4.2, we only need to show that the set of $\left(\beta_{1}, \ldots, \beta_{d}\right)$ for which the set of accumulation points of $\left(\left\{q_{n} \beta_{1}\right\}, \ldots,\left\{q_{n} \beta_{d}\right\}\right)_{n \geq 1}$ is dense in $\mathbb{T}^{d}$, is residual (i.e. it includes a dense $G_{\delta}$ subset).

We take $\varepsilon>0, c_{1}, \ldots, c_{d} \in[0,1)$ and consider the sets $\widetilde{A}_{N}=\widetilde{A}_{N}\left(c_{1}, \ldots, c_{d}, \varepsilon\right):=$ $\bigcup_{n=N}^{\infty} A_{n}\left(c_{1}, \ldots, c_{d}, \varepsilon\right)$, where

$$
A_{n}=A_{n}\left(c_{1}, \ldots, c_{d}, \varepsilon\right):=\left\{\left(\beta_{1}, \ldots, \beta_{d}\right) \in \mathbb{T}^{d}:\left\|q_{n} \beta_{1}-c_{1}\right\|<\varepsilon, \ldots,\left\|q_{n} \beta_{k}-c_{d}\right\|<\varepsilon\right\} .
$$

Clearly $\widetilde{A}_{N}$ is open and also dense. Fix $0<\varepsilon_{\ell} \rightarrow 0$. Then the set

$$
\bigcap_{\ell \geq 1} \bigcap_{N=1}^{\infty} \widetilde{A}_{N}\left(c_{1}, \ldots, c_{d}, \varepsilon_{\ell}\right)
$$

is a dense $G_{\delta}$. Moreover this set equals

$$
\left\{\left(\beta_{1}, \ldots, \beta_{d}\right) \in \mathbb{T}^{d}:\left(\exists q_{n_{k}}\right) \quad\left(\left\{q_{n_{k}} \beta_{1}\right\}, \ldots,\left\{q_{n_{k}} \beta_{d}\right\}\right) \rightarrow\left(c_{1}, \ldots, c_{d}\right)\right\}
$$

so the latter set is also a dense $G_{\delta}$. Therefore the set

$$
\bigcap_{\left(c_{1}, \ldots, c_{d}\right) \in \mathbb{Q}^{d} \cap[0,1)^{d}} \bigcap_{\ell=1}^{\infty} \bigcap_{N=1}^{\infty} \widetilde{A}_{N}\left(c_{1}, \ldots, c_{k}, \varepsilon_{\ell}\right)
$$

is a dense $G_{\delta}$ and the proof is complete.

Now, we show that the multiple ergodicity problem has a positive answer for a.a. choices of $\left(\beta_{1}, \ldots, \beta_{d}\right)$. We will need the following classical lemma of Rajchman.

Lemma 4.5. Let $(X, \mathcal{B}, \mu)$ be a probability space, $f_{n}: X \rightarrow \mathbb{R}$ such that $f_{n} \in L^{2}(X, \mathcal{B}, \mu)$, $\left\|f_{n}\right\|<C$, and $f_{n} \perp f_{m}$ whenever $n \neq m$. Then $\frac{1}{n} \sum_{k=1}^{n} f_{k} \rightarrow 0$ a.e.

Proof. It follows from the assumptions that $\sum_{N=1}^{\infty}\left\|\frac{1}{N^{2}} \sum_{k=1}^{N^{2}} f_{k}\right\|_{2}^{2} \leq \sum_{N=1}^{\infty} \frac{C^{2}}{N^{2}}<+\infty$; hence, $\lim _{N} \frac{1}{N^{2}} \sum_{k=1}^{N^{2}} f_{k}=0$ a.e.

For $n \geq 1$, let $L_{n}:=[\sqrt{n}]$. We have $L_{n}^{2} \leq n<\left(L_{n}+1\right)^{2}$ and

$$
\left|\frac{1}{n} \sum_{k=1}^{n} f_{k}\right| \leq \frac{1}{L_{n}^{2}}\left|\sum_{k=1}^{L_{n}^{2}} f_{k}\right|+2 C \frac{L_{n}}{n} \underset{n \rightarrow \infty}{\longrightarrow} 0 \text {, a.e. }
$$

Proposition 4.6. For every irrational rotation $T x=x+\alpha$ on $\mathbb{T}$, we have

$$
\left.\mu^{\otimes d}\left\{\left(\beta_{1}, \ldots, \beta_{d}\right) \in \mathbb{T}^{d}:\left(\psi, \psi \circ S_{\beta_{1}}, \ldots, \psi \circ S_{\beta_{d}}\right)\right) \text { is } T \text {-ergodic }\right\}=1 .
$$


Proof. By Corollary 4.2, all we need to show is that the set of $\left(\beta_{1}, \ldots, \beta_{d}\right)$ for which the set of accumulation points of $\left(\left\{q_{n} \beta_{1}\right\}, \ldots,\left\{q_{n} \beta_{d}\right\}\right)_{n \geq 1}$ is dense in $\mathbb{T}^{d}$, is a set of full measure. We will show more: the set of such $d$-tuples for which $\left(\left\{q_{n} \beta_{1}\right\}, \ldots,\left\{q_{n} \beta_{d}\right\}\right)_{n \geq 1}$ is uniformly distributed $(\bmod 1)$ in $\mathbb{T}^{d}$ is of full measure.

For almost all $\left(\beta_{1}, \ldots, \beta_{d}\right)$, the sequence $\left(q_{n} \beta_{1}, \ldots, q_{n} \beta_{d}\right)_{n \geq 1}$ is uniformly distributed $(\bmod 1)$. Indeed, by Weyl's criterium of equidistribution (see e.g. [KuNi]) it suffices to show that for almost all $\left(\beta_{1}, \ldots, \beta_{d}\right)$ in $\mathbb{T}^{d}$, for any nontrivial character $\chi$ of $\mathbb{T}^{d}$, the Cesaro averages of the sequence $\left(\chi\left(q_{n} \beta_{1}, \ldots, q_{n} \beta_{d}\right)\right)_{n \geq 1}$ tend to zero.

We have $\chi\left(q_{n} \beta_{1}, \ldots, q_{n} \beta_{d}\right)=\exp \left(2 \pi i\left(s_{1} q_{n} \beta_{1}+\ldots+s_{d} q_{n} \beta_{d}\right)\right)$ for integers $s_{1}, \ldots, s_{d}$. To conclude, we apply Lemma 4.5 to $f_{n}\left(x_{1}, \ldots, x_{d}\right):=\exp \left(2 \pi i\left(q_{n} s_{1} x_{1}+\ldots+q_{n} s_{d} x_{d}\right)\right)$.

The authors are grateful to M. Lemańczyk and E. Lesigne for their valuable suggestions. They thank the referee for his careful reading and his helpful remarks.

\section{References}

[Aa97] J. Aaronson, An Introduction to Infinite Ergodic Theory, Mathematical Surveys and Monographs 50, AMS, Providence, 1997.

[Bo85] M. Boshernitzan, A condition for minimal interval exchange maps to be uniquely ergodic, Duke Math. J. 52 (1985), 723-752.

[Co80] J.-P. Conze, Ergodicité d'une transformation cylindrique, Bull. Soc. Math. France 108 (1980), 441-456.

[Co09] J.-P. Conze, Recurrence, ergodicity and invariant measures for cocycles over a rotation, pp. 45-70 in Contemporary Mathematics 485, AMS, Providence, RI, 2009.

[CoFr11] J.-P. Conze, K. Frączek, Cocycles over interval exchange transformations and multivalued Hamiltonian flows, Adv. Math. 226 (2011), 4373-4428.

[CoGu12] J.-P. Conze, E. Gutkin, On recurrence and ergodicity for geodesic flows on non-compact periodic polygonal surfaces, Ergodic Theory Dynam. Systems, 32 (2012), 491-515.

[Fr00] K. Frączek, On ergodicity of some cylinder flows, Fund. Math. 163 (2000), 117-130.

[GuPa06] M. Guenais, F. Parreau, Valeurs propres de transformations liées aux rotations irrationnelles et aux fonctions en escalier, preprint 2006, arXiv 0605250v1.

[IN88] S. Ito, H. Nakada, Approximations of real numbers by the sequemce $\{$ n $\alpha\}$ and their metrical theory, Acta Math. Hung. 52 (1988), 91-100. 
[Kh] A. Khinchin, Continued Fractions, Dover Publications, Mineola, N.Y, 1997.

[KrLi91] C. Kraaikamp, P. Liardet, Good approximations and continued fractions, Proc. Amer. Math. Soc. 112 (1991), 303-309.

[KuNi] L. Kuipers, H. Niederreiter, Uniform Distribution of Sequences, Wiley, 1974.

[La88] G. Larcher, A convergence problem connected with continued fractions, Proc. Amer. Math. Soc. 103 (1988), 718-722.

[Le93] E. Lesigne, Equations fonctionnelles, couplages de produits gauches et théorèmes ergodiques pour mesures diagonales, Bull. Soc. Math, France $\mathbf{1 2 1}$ (1993), 315-351.

[LePaVo96] M. Lemańczyk, F. Parreau, D. Volný, Ergodic properties of real cocycles and pseudo-homogeneous Banach spaces, Trans. Amer. Math. Soc. 348 (1996), 4919-4938.

[LeMeNa03] M. Lemańczyk, M. Mentzen, H. Nakada, Semisimple extensions of irrational rotations, Studia Math. 156 (2003).

[Me85] K. Merrill, Cohomology of step functions under irrational rotations, Israel J. Math. 52 (1985), 320-340.

[MoSc80] C.C. Moore, K. Schmidt, Coboundaries and homomorphisms for nonsingular actions and a problem of $H$. Helson, Proc. London Math. Soc. (3) 40 (1980), 443-475.

[Or83] I. Oren, Ergodicity of cylinder flows arising from irregularities of distribution, Israel J. Math. 44 (1983), 127-138.

[Pa90] D. Pask, Skew products over irrational rotation, Israel J. Math. 69 (1990), 65-74.

[Sc77] K. Schmidt, Lectures on Cocycles of Ergodic Transformations Groups, Lect. Notes in Math. vol. 1, Mac Millan Co. of India (1977).

[Zh10] Y. Zhang, Ergodicity of $\mathbb{Z}^{2}$ extensions of irrational rotations, Studia Math. 204 (2011), 235-246.

Jean-Pierre Conze

IRMAR, CNRS UMR 6625, University of Rennes I, Campus de Beaulieu, 35042 Rennes Cedex, France

conze@univ-rennes1.fr

Agata Piękniewska

Faculty of Mathematics and Computer Science,

Nicolaus Copernicus University,

ul. Chopina 12/18, 87-100 Toruń, Poland

a.piekniewska@gmail.com 